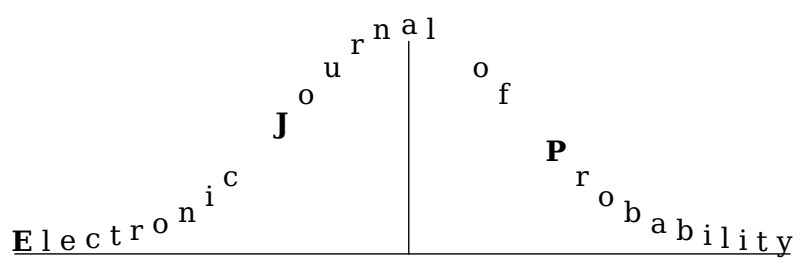

Electron. J. Probab. 27 (2022), article no. 31, 1-26.

ISSN: 1083-6489 https://doi.org/10.1214/21-EJP737

\title{
Rate of escape of conditioned Brownian motion*
}

\author{
Orphée Collin $^{\dagger} \quad$ Francis Comets ${ }^{\ddagger}$
}

\begin{abstract}
We study the norm of the two-dimensional Brownian motion conditioned to stay outside the unit disk at all times. By conditioning the process is changed from barely recurrent to slightly transient. We obtain sharp results on the rate of escape to infinity of the process of future minima:

(i) we find an integral test on the function $g$ so that the future minima process drops below the barrier $\exp \{\ln t \times g(\ln \ln t)\}$ at arbitrary large times;

(ii) we show that the future minima process exceeds $K \sqrt{t \times \ln \ln \ln t}$ at arbitrary large times with probability 0 [resp., 1] if $K$ is larger [resp., smaller] than some positive constant.
\end{abstract}

For this, we introduce a renewal structure attached to record times and values. Additional results are given for the long time behavior of the norm.

Keywords: Brownian motion; Bessel process; conditioning; transience; Wiener moustache; regeneration; upper-class and lower-class; random difference equation; autoregressive process. MSC2020 subject classifications: 60K35; 60J60; 60J65; 60G17.

Submitted to EJP on April 4, 2021, final version accepted on December 30, 2021.

Supersedes arXiv: 2102.09636.

Supersedes HAL : hal - 03146283.

\section{Introduction}

This paper is devoted to the planar Brownian motion conditioned to stay outside the unit ball $\mathrm{B}(0,1)$ at all times. Besides its own appeal from its fundamental character, this process has attracted a keen interest as being the elementary brick of the twodimensional Brownian random interlacement recently introduced in [9]. By rotational symmetry, the norm $R$ of the conditioned Brownian motion itself follows a stochastic differential equation in $[1, \infty)$,

$$
d R(t)=\left(\frac{1}{R(t) \ln R(t)}+\frac{1}{2 R(t)}\right) d t+d B(t)
$$

*Francis Comets is partially supported by ANR SWIWS.

${ }^{\dagger}$ DMA, École normale supérieure, Université PSL, CNRS, 75005 Paris, France.

E-mail: orphee.collin@normalesup.org

‡Université de Paris and LPSM, Mathématiques, case 7012, F-75205 Paris Cedex 13, France.

E-mail: comets@lpsm.paris 
with $B$ a standard Brownian motion in $\mathbb{R}$, and we can - and we will - restrict the study of the conditioned process to that of $R$ itself since the angle obeys a diffusion subordinated to it. The two-dimensional Brownian motion is critically recurrent, but conditioning it outside the unit ball turns it into (delicately) transient. A natural question is the rate at which $R(t)$ tends to $\infty$ as $t \rightarrow \infty$, this is the object of the present paper. A measure of the reluctance of $R$ to tend to infinity is given by the future minima process

$$
M(t)=\inf \{R(s) ; s \geq t\}
$$

which is non-decreasing to $\infty$ a.s. The corresponding model in the discrete case, the two-dimensional simple random walk conditioned to avoid the origin at all times, has motivated many recent papers. Estimates on the future minimum distance to the origin have been obtained in [22], we will use them as benchmarks. It is also shown that two independent conditioned walkers meet infinitely often although they are transient. The range of the walk, i.e. the set of visited sites, is studied in [11]: if a finite $A \subset \mathbb{Z}^{2} \backslash\{0\}$ is "big enough and well distributed in space", then the proportion of visited sites is approximately uniformly distributed on $[0,1]$. In [20] the explicit formula for the Green function is obtained, and a survey is given in Chapter 4 of [21].

For dimensions $d \geq 3$, the random interlacement model has been introduced in [27] to describe the local picture of the visited set by a random walk at large times on a large $d$-dimensional torus, and similarly in [28], the Brownian random interlacement to describe the Wiener sausage around the Brownian motion on a $d$-dimensional torus. For dimension $d=2$, the random interlacement model is the local limit of the visited set by the random walk around a point which has not been visited so far [7], and analogously, the Brownian random interlacement is the local limit of the Wiener sausage on the two-dimensional torus around a point which is outside the sausage [9]. Formally, the two-dimensional Brownian random interlacement is defined as a Poisson process of bi-infinite paths, which are rescaled instances of the so-called "Wiener moustache". The Wiener moustache is obtained by gluing two instances (for positive and negative times, see Figure 1 in [9]) of planar Brownian motion conditioned to stay outside the unit ball, which are independent except that they share the same starting point (see Lemma 3.9 in [9]). Hence, the process we consider in this paper is the building brick of Brownian random interlacement in the plane. We also recall that the complement of the sausage around the interlacement has an interesting phase transition, changing from a.s. unbounded to a.s. bounded as the Poisson intensity is increased, see Th. 2.13 in [9] and [8] for the discrete case.

With a slight abuse of terminology, we say $f(t) \leq g(t)$ i.o. (infinitely often) if the set $\{t \geq 0: f(t) \leq g(t)\}$ is unbounded, and $f(t) \leq g(t)$ ev. (eventually) if the set $\{t \geq 0: f(t) \leq g(t)\}$ is a neighborhood of $\infty$ in $\mathbb{R}_{+}$.

We now give a short overview of some of our results on the rate of escape of $R$ to infinity. They are consequences of the results in section 2.1.

Theorem 1.1. For $g: \mathbb{R}_{+} \rightarrow \mathbb{R}_{+}$non-increasing such that $(\ln t) g(\ln \ln t)$ is non-decreasing,

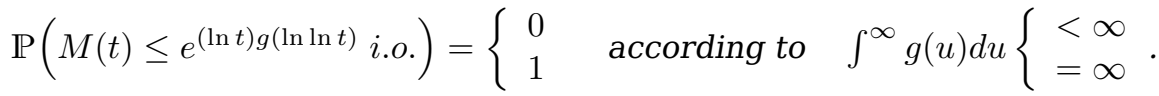

This result with an integral condition has a flavor of Kolmogorov's test (see, e.g., sect. 4.12 in [14]).

Theorem 1.2. The limit

$$
K^{*}=\limsup _{t \rightarrow \infty} \frac{M(t)}{\sqrt{t \ln \ln \ln t}}
$$

is almost surely constant, and

$$
0<K^{*}<\infty
$$


Though we do not know the actual value of $K^{*}$ we can see that both theorems are much finer than the corresponding Theorem 1.2 of [22]. These two theorems together yield a precise version of the observation from [20] that the pathwise divergence of $R$ to infinity occurs in a highly irregular way. The future minima process has been considered earlier, e.g. [16] and [17] for Bessel processes and for random walks, and [19] for positive self-similar Markov processes. Let us recall the similar result for transient Bessel processes. Denote by $\mathrm{BES}^{d}$ the $d$-dimensional Bessel process, i.e. the solution of the stochastic differential equation

$$
d X(t)=\frac{d-1}{2 X(t)} d t+d B(t)
$$

that is the norm of the standard Brownian motion in $\mathbb{R}^{d}$ when $d$ integer: then, by Th. 4.1 in [16],

$$
\text { for } d>2, \quad \limsup _{t \rightarrow \infty} \frac{\min \left\{\operatorname{BES}^{d}(s) ; s \geq t\right\}}{\sqrt{2 t \ln \ln t}}=1 .
$$

An important (and beautiful) finding of our work is a renewal structure in Section 3 which allows sharp estimates. To illustrate that, let's mention that we will find a sequence of relevant random variables $S_{n}>0$ solving a random difference equation

$$
S_{n}=\alpha_{n} S_{n-1}+\beta_{n}, \quad n \geq 1
$$

where the sequence $\left(\alpha_{n}, \beta_{n}\right)_{n}$ is i.i.d. with positive coefficients, $\alpha_{n}<1$ and $\beta_{n}$ with logarithmic tails, $\mathbb{P}\left(\beta_{1}>t\right) \sim c / \ln t$ for large $t$. Although autoregressive processes $\operatorname{AR}(1)$ of the type (1.4) are usually addressed with exponential or power-law tail for $\beta_{n}$, see [5], the case of logarithmic tail has been also considered, see [15], [31], [3], and also both papers [1] and [32] for a recent account. Interestingly, our model is critical in the perspective of the Markov chain $S_{n}$, in the sense that the actual value of the constant $c$ is precisely the transition from recurrence to transience for the chain.

The paper is organized as follows. We give the main results in the next section. The regeneration structure is defined in Section 3, together with the basic estimates, and ending with Remark 3.8 on the above random difference equation. In the next section we prove some results showing that $R$ somewhat behaves at large times like the two-dimensional Bessel process. In Sections 5 and 6 we prove the two above theorems.

\section{Main results}

We first collect a few properties of the involved processes.

We start with some notations. Consider $W$ a two-dimensional standard Brownian motion and denote by $\mathrm{P}_{x}$ the law of $W$ starting at $x, \widehat{W}$ a Brownian motion conditioned to stay outside the unit ball, and denote by $\widehat{\mathrm{P}}_{x}$ its law when starting at $x$, and $R=|\widehat{W}|$ its Euclidean norm with $P_{r}$ the corresponding law $(r=|x|)$. In this paper we are mainly interested in $\mathbb{P}=P_{1}$. The construction of the process starting from $R(0)>1$ is standard from taboo process theory, and the one starting from $R(0)=1$ is given in definition 2.2 of [9].

Denote by $|\cdot|$ the Euclidean norm and $\mathrm{B}(x, r)$ the closed ball with center $x$ and radius $r>0$. For a closed subset $B$ of the state space of a process $Y$, we denote the entrance time $\tau(Y ; B)=\inf \{t \geq 0: Y(t) \in B\}$, and write for short $\tau(Y ; r)=\tau(Y ; \partial \mathrm{B}(0, r))$ and also $\tau(r)=\tau(R ; r)$ when $Y=R$. The function $h(x)=\ln |x|$ is harmonic in $\mathbb{R}^{2} \backslash\{0\}$, positive on $\mathbb{R}^{2} \backslash \mathrm{B}(0,1)$ and vanishes on the unit circle. Then, the law $\widehat{\mathrm{P}}_{x}$ of the planar Brownian motion $W$ conditioned outside $\mathrm{B}(0,1)$ is given by Doob's $h$-transform of $\mathrm{P}_{x}$. By definition, 
for $A \subset \mathcal{C}\left(\mathbb{R}^{+}, \mathbb{R}\right)$ which is $\mathcal{F}_{\tau\left(r_{1}\right)}$-measurable $\left(1<|x|=r<r_{1}\right)$,

$$
\begin{aligned}
P_{r}(R \in A) & =\mathrm{P}_{x}\left(|W| \in A \mid \tau\left(W ; r_{1}\right)<\tau(W ; 1)\right) \\
& =\mathrm{P}_{x}\left(|W| \in A, \tau\left(W ; r_{1}\right)<\tau(W ; 1)\right) \times \frac{\ln r_{1}}{\ln |x|},
\end{aligned}
$$

recalling that $\mathrm{P}_{x}\left(\tau\left(W ; r_{1}\right)<\tau(W ; 1)\right)=\frac{\ln |x|}{\ln r_{1}}$ since $\ln |x|$ is harmonic in $\mathbb{R}^{2} \backslash\{0\}$.

Another remarkable property is Remark 3.8 in [9]: For all $x \notin \mathrm{B}(1), \rho>0$, we have

$$
\widehat{\mathrm{P}}_{x}[\tau(\widehat{W} ; \mathrm{B}(y, \rho))<\infty] \rightarrow \frac{1}{2} \quad \text { as }|y| \rightarrow \infty \text {. }
$$

The scale function for the process $R$ - that is, the unique (up to affine transformation) real function $S$ such that $S(R(t))$ is a local martingale - is $S(r)=\frac{-1}{\ln r}$. Then, for $1<a<r<b$,

$$
P_{r}[\tau(b)<\tau(a)]=\frac{\ln (r / a) \times \ln b}{\ln (b / a) \times \ln r} .
$$

We refer to section 2.1 in [9] for more details on the many interesting properties of $\widehat{W}$ and $R$.

\subsection{Results for the future minimum}

With $L(t)=\ln (t \vee 1)$ and $\ln (\cdot)$ the natural logarithm, define $\ln _{1}(t)=L(t)$, and for $k \geq 2, \ln _{k}(t)=L\left(\ln _{k-1}(t)\right)$ so that $\ln _{k}(t)=(\ln \circ \ldots \circ \ln )(t)$ for $t$ large.

Theorem 2.1. For $g: \mathbb{R}_{+} \rightarrow \mathbb{R}_{+}$non-increasing such that $(\ln t) g\left(\ln _{2} t\right)$ is non-decreasing, we have:

$$
\int^{\infty} g(u) d u<\infty \Longrightarrow \text { a.s., } M(t) \geq e^{(\ln t) g\left(\ln _{2} t\right)} \quad \text { eventually }
$$

and

$$
\int^{\infty} g(u) d u=\infty \Longrightarrow \text { a.s., } M(t) \leq e^{(\ln t) g\left(\ln _{2} t\right)} \quad \text { infinitely often. }
$$

(Note that the second assumption is quite natural in view of the monotonicity of $M(t)$.) Theorem 1.1 is a direct consequence of the above theorem. This result with an integral condition is reminiscent of Kolmogorov's test (see, e.g., sect. 4.12 in [14]), but the process $M$ here is not Markov.

These estimates are stronger than the corresponding ones in Th. 1.2 of [22]. So are the following ones:

Theorem 2.2. There exist $0<K^{\prime}<K<\infty$ such that, almost surely,

$$
M(t) \leq K \sqrt{t \ln _{3} t} \text { eventually }
$$

and

$$
M(t) \geq K^{\prime} \sqrt{t \ln _{3} t} \quad \text { infinitely often } .
$$

Theorem 1.2 is essentially a reformulation of Theorem 2.2, it will be proved below Remark 6.2.

We recall the similar result (1.3) for transient Bessel processes: a.s. for all $a<\sqrt{2}<b$, the future minima process $\min \left\{\operatorname{BES}^{d}(s) ; s \geq t\right\}$ is eventually smaller than $b \sqrt{t \ln _{2} t}$ and infinitely often larger than $a \sqrt{t \ln _{2} t}$.

Finally we mention that, for $d>2, \min \left\{\operatorname{BES}^{d}(s) ; s \geq t\right\} \leq \varepsilon \sqrt{t \ln _{2} t}$ i.o., a.s. for all $\varepsilon>0$. (See [16], P.349.) 


\subsection{Long time behavior of $R(t)$}

At large times the process $R$ behaves like $\mathrm{BES}^{2}$. We emphasize that this is for the marginal law, but not for the future minimum. We formulate here precise statements of these facts.

It is well known that the random variable $t^{-1 / 2} \operatorname{BES}^{2}(t)$ converges to the Rayleigh distribution

$$
d \nu(x)=x e^{-x^{2} / 2} \mathbf{1}_{(0, \infty)}(x) d x
$$

as $t \rightarrow \infty$. Similarly for $R$, we have

Theorem 2.3. Let $Z \sim \nu$. As $t \rightarrow \infty$,

$$
\frac{R(t)}{\sqrt{t}} \stackrel{\text { law }}{\longrightarrow} Z
$$

Theorem 2.4 (Pointwise ergodic theorem). For all bounded continuous function $f$ on $(0, \infty)$, as $t \rightarrow \infty$,

$$
\frac{1}{t} \int_{0}^{e^{t}-1} f\left(\frac{R(u)}{\sqrt{1+u}}\right) \frac{1}{1+u} d u \longrightarrow \int_{\mathbb{R}} f d \nu \quad \text { a.s. }
$$

We will prove Theorems 2.3 and 2.4 in section 4 .

\section{Regenerative structure}

We fix a parameter $r>1$. We construct a regenerative structure associated with the process $R$ starting from $R(0)=1$.

\subsection{Renewal times}

We define a random sequence $\left(H_{n}, A_{n}, T_{n}\right)_{n \geq 0}$ by $H_{0}, T_{0}=0, A_{0}=1$, then

$$
\left\{\begin{array}{l}
H_{1}=\inf \left\{t>T_{0}: R(t)=r\right\} \\
A_{1}=\inf \left\{R(t) ; t \geq H_{1}\right\} \\
T_{1}=\inf \left\{t \geq H_{1}: R(t)=A_{1}\right\}
\end{array}\right.
$$

and for $n \geq 1$,

$$
\left\{\begin{array}{l}
H_{n+1}=\inf \left\{t>T_{n}: R(t)=r A_{n}\right\} \\
A_{n+1}=\inf \left\{R(t) ; t \geq H_{n+1}\right\} \\
T_{n+1}=\inf \left\{t \geq H_{n+1}: R(t)=A_{n+1}\right\} .
\end{array}\right.
$$

Since $R$ is a continuous function with $\lim _{t \rightarrow \infty} R(t)=\infty$ a.s., we see by induction that $T_{n}<\infty$ a.s. with $T_{n}<T_{n+1}$ and $\lim _{n \rightarrow \infty} T_{n}=\infty$ a.s. The $T_{n}$ are not stopping times, but they are called renewal times for the following reasons.

Proposition 3.1. Let $\mathcal{G}_{1}=\sigma\left(T_{1},\left(R(t) \mathbb{1}_{t<T_{1}} ; t \geq 0\right)\right)$. Then,

$$
\left(\frac{R\left(T_{1}+A_{1}^{2} t\right)}{A_{1}} ; t \geq 0\right)
$$

has same law as $R$ and is independent of $\mathcal{G}_{1}$.

This proposition is the building brick of the

Theorem 3.2 (Renewal structure). The sequence

$$
\left(\frac{R\left(T_{n}+A_{n}^{2} t\right)}{A_{n}} ; t \in\left[0, \frac{T_{n+1}-T_{n}}{A_{n}^{2}}\right]\right)_{n \geq 0}
$$

is independent and identically distributed with the law of $\left(R(t) ; t \in\left[0, T_{1}\right]\right)$. 
In particular, since $R\left(T_{n+1}\right)=A_{n+1}$, the sequence

$$
\left(\frac{T_{n+1}-T_{n}}{A_{n}^{2}}, \frac{A_{n+1}}{A_{n}}\right)_{n \geq 0}
$$

is i.i.d. and distributed as $\left(T_{1}, A_{1}\right)$. Therefore $\left(T_{n}, A_{n}\right)$ can be written using i.i.d.r.v.'s, which will be used repeatedly all through.

Proof. Proposition 3.1. Recall that $P_{r}$ denotes the law of the process $R$ with $R(0)=r$. Observe that $H_{1}$ is a stopping time, and denote by $\mathcal{F}_{H_{1}}$ the sigma-field of events that occur before time $H_{1}$. By the strong Markov property,

$$
\text { under } P_{1},\left(R\left(H_{1}+t\right)\right)_{t \geq 0} \text { is independent of } \mathcal{F}_{H_{1}} \text { and has the law } P_{r} \text {. }
$$

Moreover, by Theorem 2.4 in [30] (see also the proof of Lemma 3.9 in [9]), conditionally on $T_{1},\left(R(t) ; t \in\left[H_{1}, T_{1}\right]\right)$ and $A_{1}=a,\left(R\left(T_{1}+t\right) ; t \geq 0\right)$ has the same law as $R$ starting from $a$ and conditioned to $R(t) \geq a, \forall t \geq 0$. By Brownian scaling, the latter law is equal to that of $a R\left(\cdot / a^{2}\right)$ under $P_{1}$; see also Remark 2.5 in [9]. Since $\mathcal{G}_{1}=\sigma\left(\mathcal{F}_{H_{1}} ;\left(R(t) ; t \in\left[H_{1}, T_{1}\right]\right)\right)$ up to null events, we obtain the desired statement.

Proof. Theorem 3.2. By induction, Proposition 3.1 implies that for all $n$, the process $\left(\frac{R\left(T_{n}+A_{n}^{2} t\right)}{A_{n}} ; t \geq 0\right)$ is independent of $\mathcal{G}_{n}=\sigma\left(T_{n},\left(R(t) ; t<T_{n}\right)\right)$ with the law of $R$. Then, the claim follows.

As a direct consequence we have discovered a simple representation of crucial times and points of the process.

Corollary 3.3. Define

$$
A_{n+1}^{\prime}=\frac{A_{n+1}}{A_{n}}, \quad T_{n+1}^{\prime}=\frac{T_{n+1}-T_{n}}{A_{n}^{2}}, \quad n \geq 0 .
$$

Then, $\left(A_{n}^{\prime}, T_{n}^{\prime}\right)_{n \geq 1}$ is an i.i.d. sequence with the same law as $\left(A_{1}, T_{1}\right)$, and we have the representation

$$
\left\{\begin{array}{l}
T_{n}=T_{1}^{\prime}+A_{1}^{\prime 2} T_{2}^{\prime}+\ldots+\left(A_{1}^{\prime} \ldots A_{n-1}^{\prime}\right)^{2} T_{n}^{\prime} \quad, \quad n \geq 1 . \\
A_{n}=A_{1}^{\prime} \ldots A_{n}^{\prime}
\end{array}\right.
$$

\subsection{Description of a cycle}

Recall $r>1$ is fixed. We will shorten the notations: $(H, A, T)=\left(H_{1}, A_{1}, T_{1}\right)$. Recall that $R$ starts from $R(0)=1$, hits $r$ at $H$ for the first time, and reaches its future minimum $A \in(1, r)$ at time $T$. We also introduce its maximum $B>r$ on the time interval $[H, T]$, as well as their logarithms $U, V$ :

$$
\left\{\begin{array}{l}
A=r^{U}=\min \{R(t) ; t \geq H\} \\
B=r^{V}=\max \{R(t) ; t \in[H, T]\}
\end{array}\right.
$$

see figure 1. It was shown in [9] that $U$ is uniform on [0,1] (see (2.2) with $b \rightarrow \infty$ ), but we can even compute the joint law of $U$ and $V$. For $1<a-h<a<r<b$, we have by the strong Markov property

$$
\begin{aligned}
\mathbb{P}(A & \in[a-h, a), B>b) \\
& =\mathbb{P}(A \in[a-h, a), B>b, \tau(b)<\tau(a))+\mathbb{P}(A \in[a-h, a), B>b, \tau(b)>\tau(a)) \\
& =P_{r}(\tau(b)<\tau(a)) \times P_{b}(\min \{R(t) ; t \geq 0\} \in[a-h, a))+o(h) \\
& =\frac{\ln (r / a) \ln b}{\ln (b / a) \ln r} \times \frac{1}{a \ln b} h+o(h),
\end{aligned}
$$




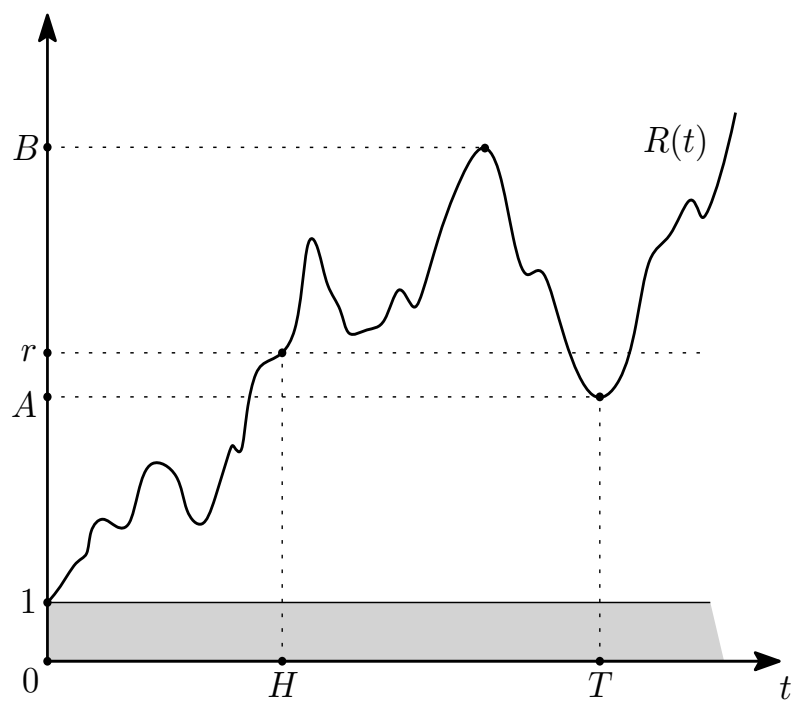

Figure 1: First cycle: $A=r^{U}, B=r^{V}$

using (2.16) in [9] and that, for $R$ started at $b, \min \{R(t) ; t \geq 0\}$ has density $(a \ln b)^{-1}$ on $(1, b)$. Hence $(A, B)$ has a density given by the negative of the $b$-derivative of the dominant term as $h \searrow 0$, i.e.,

$$
p_{A, B}(a, b)=\frac{1}{a b \ln r} \frac{\ln (r / a)}{\ln ^{2}(b / a)}, \quad 1<a<r<b .
$$

By changing variables, it follows that $(U, V)$ has density

$$
p_{U, V}(u, v)=\frac{1-u}{(v-u)^{2}} \mathbb{1}_{0<u<1<v} .
$$

We recover that $U$ is uniform on $(0,1)$ and that $V$ has density

$$
p_{V}(v)=-\ln (1-1 / v)-1 / v, \quad v>1 .
$$

It follows that for $v \geq 1$,

$$
\mathbb{P}(V>v)=\sum_{n=1}^{\infty} \frac{1}{n(n+1) v^{n}},
$$

and then $\mathbb{P}(V>v) \sim 1 /(2 v)$ as $v \rightarrow \infty$.

We also need information on the cycle length $T$. For any $s \geq 1$ we consider the hitting time by $R$ starting at $s$ of its absolute minimum, and denote by $\mu_{s}$ a r.v. with the same law:

$$
\mu_{s} \sim P_{s}(\arg \min \{R(t) ; t \geq 0\} \in \cdot) .
$$

Recall that, under $\mathbb{P}, R(0)=1$.

Proposition 3.4. (i) We have

$$
T=H+(T-H),
$$

where $H$ and $(T-H)$ are independent with $T-H \stackrel{\text { law }}{=} \mu_{r}$.

(ii) For $u \in(0,1)$, the conditional law of $T$ given $U \geq u$ is equal to the law of an independent sum $H+r^{2 u} \mu_{\left(r^{1-u}\right)}$. 
Proof. (i) directly follows from the strong Markov property for the Markov process $R$ and the stopping time $H$.

For (ii), we recall Remark 2.5 in [9]: for $c>1$, denoting by $R^{c}$ the diffusion $R$ conditioned to stay outside $(1, c]$, and started at $r \geq c$, we have

$$
R^{c}(\cdot)=c R\left(\cdot / c^{2}\right) \quad \text { in law }
$$

with $R$ started at $r / c$. (Alternatively, this follows from $R$ being the norm of conditioned Brownian motion (2.1) and from Brownian scaling.) Hence, for $s \in \mathbb{R}$, again from the strong Markov property,

$$
\begin{aligned}
E_{1}\left[e^{i s T} \mid U \geq u\right] & =E_{1}\left[e^{i s(T-H+H)} \mid U \geq u\right] \\
& =E_{1}\left[e^{i s H}\right] \times E_{r}\left[e^{i s(T-H)} \mid U \geq u\right] \\
& =E_{1}\left[e^{i s H}\right] \times E_{r}\left[e^{i s \times \arg \min \{R(t) ; t \geq 0\}} \mid \min \{R(t) ; t \geq 0\} \geq r^{u}\right] \\
& =E_{1}\left[e^{i s H}\right] \times E\left[e^{i s r^{2 u} \mu_{\left(r^{1}-u\right)}}\right],
\end{aligned}
$$

which proves the result.

\subsection{Tail estimates for $T$}

We need some estimates of the upper and lower tails of $T$, that we derive in this section. But first we state elementary comparisons of $R$ and Bessel processes, see (1.2), that will be used all through the paper.

Proposition 3.5. (i) There exists a coupling of the processes $R$ and $\mathrm{BES}^{2}$ starting at 1 such that

$$
\forall t \geq 0, \quad R(t) \geq \operatorname{BES}^{2}(t) .
$$

(ii) For $\delta>0$ there exists a coupling of the processes $R$ and $\mathrm{BES}^{2+\delta}$ starting at 1 such that for $\sigma=\sup \left\{t \geq 0 ; R(t) \leq e^{2 / \delta}\right\}$,

$$
\forall s \geq 0, \quad R(\sigma+s) \leq \operatorname{BES}^{2+\delta}(\sigma+s)-\operatorname{BES}^{2+\delta}(\sigma)+e^{2 / \delta} .
$$

Proof. It is well known [6] that the stochastic differential equation (1.2) has a strong solution, so we can couple the processes $R$ and $\mathrm{BES}^{2}, \mathrm{BES}^{2+\delta}$ by driving equations (1.1) and (1.2) by the same Brownian motion $B$. Then, with $x^{+}=\max \{x, 0\}$ for $x$ real, we have for all $t>0$ and all realization of $B$,

$$
\begin{aligned}
d\left(\operatorname{BES}^{2}(t)-R(t)\right)^{+} & =\mathbb{1}_{\left\{\operatorname{BES}^{2}(t) \geq R(t)\right\}}\left(\frac{1}{2 \operatorname{BES}^{2}(t)}-\frac{1}{2 R(t)}-\frac{1}{R(t) \ln R(t)}\right) d t \\
& \leq 0,
\end{aligned}
$$

which implies (i) by integration. Similarly for (ii) we write the differential

$$
\begin{aligned}
d\left(R(t)-\mathrm{BES}^{2+\delta}(t)\right)^{+} & =\mathbb{1}_{\left\{\mathrm{BES}^{2+\delta}(t) \leq R(t)\right\}}\left(\frac{1}{2 R(t)}+\frac{1}{R(t) \ln R(t)}-\frac{1+\delta}{2 \mathrm{BES}^{2+\delta}(t)}\right) d t \\
& \leq 0 \quad \text { for } t \geq \sigma .
\end{aligned}
$$

Integrating on $t \in[\sigma, \sigma+s]$ we obtain (ii).

We are now ready to start with the upper tail of $T$.

Proposition 3.6. As $t \rightarrow \infty$,

$$
\mathbb{P}(T \geq t) \sim \frac{\ln r}{\ln t} .
$$

More precisely, there exists constants $t_{0}$ and $C$ such that for all $t \geq t_{0}$,

$$
\left(1-\frac{\ln _{3} t+C}{\ln t}\right) \frac{\ln r}{\ln t} \leq \mathbb{P}[T \geq t] \leq\left(1+\frac{\ln _{3} t+C}{\ln t}\right) \frac{\ln r}{\ln t} .
$$


Proof. We first obtain two preliminary estimates.

Upper bound: for $0<\varepsilon<1$,

$$
\begin{aligned}
\mathbb{P}(T \geq t) & =\mathbb{P}\left(T \geq t, V \geq \frac{\ln t}{2(1+\varepsilon) \ln r}\right)+\mathbb{P}\left(T \geq t, V<\frac{\ln t}{2(1+\varepsilon) \ln r}\right) \\
& \leq \mathbb{P}\left(V \geq \frac{\ln t}{2(1+\varepsilon) \ln r}\right)+\mathbb{P}\left(R(s) \leq t^{\frac{1}{2(1+\varepsilon)}}, s \in[0, t]\right) \\
& \leq \frac{(1+\varepsilon) \ln r}{\ln t}+\frac{1}{5}\left(\frac{2(1+\varepsilon) \ln r}{\ln t}\right)^{2}+C_{0} \exp \left(-C_{1} t^{\varepsilon /(1+\varepsilon)}\right)
\end{aligned}
$$

for $t \geq t_{1}$ with $t_{1}>0$ not depending on $\varepsilon \in(0,1)$. Indeed, to obtain the first term we have used (3.2) in the form of $\mathbb{P}(V \geq v) \leq(1 / 2 v)+\left(1 / 5 v^{2}\right)$ for large $v$. In order to obtain the second one, we first bound $R(\cdot) \geq \operatorname{BES}^{2}(\cdot)$, with $\mathrm{BES}^{2}$ started at 0 using Proposition 3.5, and finally that there exist positive $C_{0}, C_{1}$ such that

$$
\forall t>0, \forall \rho>0, \quad \mathbb{P}\left(\operatorname{BES}^{2}(s) \leq \rho, s \in[0, t]\right) \leq C_{0} \exp \left(-C_{1} \frac{t}{\rho^{2}}\right)
$$

see e.g. exercise 1 p.106 in [26].

Lower bound: for $0<\varepsilon<1 / 2$,

$$
\begin{aligned}
\mathbb{P}(T \geq t) & \geq \mathbb{P}\left(T-H \geq t, V \geq \frac{\ln t}{2(1-\varepsilon) \ln r}\right) \\
& =\mathbb{P}\left(V \geq \frac{\ln t}{2(1-\varepsilon) \ln r}\right)-\mathbb{P}\left(T-H \leq t, V \geq \frac{\ln t}{2(1-\varepsilon) \ln r}\right) \\
& \geq \mathbb{P}\left(V \geq \frac{\ln t}{2(1-\varepsilon) \ln r}\right)-P_{r}\left(\tau\left(R ; t^{\frac{1}{2(1-\varepsilon)}}\right) \leq t\right) \\
& \geq \frac{(1-\varepsilon) \ln r}{\ln t}-C_{2} \exp \left(-C_{3} t^{\varepsilon /(1-\varepsilon)}\right)
\end{aligned}
$$

for $t \geq t_{2}$, with $t_{2}>0$ not depending on $\varepsilon \in\left(0, \frac{1}{2}\right)$. In (3.6) we have used (3.2) for the first term, and we give details for the second one: for $|x|=r>1$ by (2.1), we get for all $t>1$,

$$
\begin{aligned}
P_{r}\left(\tau\left(R ; t^{\frac{1}{2(1-\varepsilon)}}\right) \leq t\right) & =\mathrm{P}_{x}\left(\tau\left(|W| ; t^{\frac{1}{2(1-\varepsilon)}}\right) \leq t \mid \tau\left(|W| ; t^{\frac{1}{2(1-\varepsilon)}}\right)<\tau(|W| ; 1)\right) \\
& \leq \mathrm{P}_{x}\left(\tau\left(|W| ; t^{\frac{1}{2(1-\varepsilon)}}\right) \leq t\right) \times \frac{\ln t}{2(1-\varepsilon) \ln r} \\
& \leq C_{2} \exp \left(-C_{3} t^{\varepsilon /(1-\varepsilon)}\right)
\end{aligned}
$$

for some constants $C_{2}, C_{3}>0$ by the moderate deviation principle for Brownian motion.

For both the upper and lower bounds, we now choose

$$
\varepsilon=\varepsilon_{t}=\frac{\ln _{3} t+C_{4}}{\ln t}
$$

with a constant $C_{4}$. Provided the constant $C_{4}$ is large enough, the terms

$$
C_{0} \exp \left(-C_{1} t^{\varepsilon_{t} /\left(1+\varepsilon_{t}\right)}\right) \quad \text { and } \quad C_{2} \exp \left(-C_{3} t^{\varepsilon_{t} /\left(1-\varepsilon_{t}\right)}\right)
$$

are dominated by $(\ln t)^{-2}$. We then get (3.4) from (3.5) and (3.6), taking any $C$ larger than $C_{4}+\frac{4 \ln r}{5}$.

Finally, (3.3) is a direct consequence of (3.4). The proof is complete.

We also need to control the lower tail of $T$. 
Proposition 3.7. (i) For all $\varepsilon \in(0, r-1)$, there exists $t_{0}>0$ such that for $t \leq t_{0}$,

$$
\mathbb{P}[T \leq t] \leq \exp \left(-\frac{(r-1-\varepsilon)^{2}}{2 t}\right) .
$$

(ii) For all $\varepsilon>0$, there exists $t_{1}>0$ such that for $t \leq t_{1}$, and all $u \in[0,1)$,

$$
\mathbb{P}[T \leq t \mid U \geq u] \geq \exp \left(-\frac{(r-1+\varepsilon)^{2}}{2 t}\right)
$$

Proof. (i) Setting $a=1+\varepsilon / 2 \in(1, r)$ and using the strong Markov property for the hitting time of $a$ by $R$, we obtain

$$
\begin{aligned}
\mathbb{P}(T \leq t) & \leq P_{1}(\tau(r)-\tau(a) \leq t) \\
& =P_{a}(\tau(r) \leq t) \\
& \stackrel{(2.1)}{=} \mathrm{P}_{(a, 0)}(\tau(|W| ; r) \leq t \mid \tau(|W| ; r)<\tau(|W| ; 1)) \\
& \leq \mathrm{P}_{(a, 0)}(\tau(|W| ; r) \leq t) \times \frac{\ln r}{\ln a} .
\end{aligned}
$$

Recalling large deviation results for Brownian motion in small time, e.g. section 6.8 of Ch. 5 in [2],

$$
\lim _{t \rightarrow 0} t \ln \mathrm{P}_{(a, 0)}(\tau(|W| ; r) \leq t)=-\frac{(r-a)^{2}}{2},
$$

we see that the above upper bound implies (i).

(ii) Let $t \leq 1$. By Proposition 3.4-(ii), and by comparing $R$ and $\mathrm{BES}^{2}$ from Proposition 3.5 (i), we obtain

$$
\begin{aligned}
\mathbb{P}(T \leq t \mid U \geq u) & \geq \mathbb{P}\left(H \leq t-t^{2}\right) \times \mathbb{P}\left(r^{2 u} \mu_{\left(r^{1-u}\right)} \leq t^{2}\right) \\
& \geq \mathbb{P}\left(\operatorname{BES}^{2}\left(t-t^{2}\right) \geq r\right) \times \mathbb{P}\left(\mu_{\left(r^{1-u}\right)} \leq \frac{t^{2}}{r^{2 u}}\right) \\
& =\mathbb{P}_{(1,0)}\left(\left|W\left(t-t^{2}\right)\right| \geq r\right) \times P_{r^{1-u}}(\arg \min \{R(s) ; s \geq 0\} \leq \theta)
\end{aligned}
$$

with $\theta=\frac{t^{2}}{r^{2 u}}$. We estimate the first term using again large deviation for Brownian motion in small time [2]: for $|x|<r$,

$$
\lim _{t \rightarrow 0} t \ln \mathrm{P}_{x}(|W|(t) \geq r)=-\frac{(r-|x|)^{2}}{2} .
$$

To estimate the second term in (3.10), note that $R(\theta) \geq r^{1-u}+\sqrt{\theta}$ and $R(s) \geq r^{1-u}$ for all $s \geq \theta$ implies that, $P_{r^{1-u}}$-a.s., $R$ achieves its minimum before time $\theta$. Hence, by Markov property and (2.2),

$$
\begin{aligned}
P_{r^{1-u}}(\arg \min \{R(s) ; s \geq 0\} \leq \theta) & \geq P_{r^{1-u}}\left(R(\theta) \geq r^{1-u}+\sqrt{\theta}\right) \times\left(1-\frac{\ln r^{1-u}}{\ln \left(r^{1-u}+\sqrt{\theta}\right)}\right) \\
& \geq \mathbb{P}(B(\theta) \geq \sqrt{\theta}) \times\left(1-\frac{\ln r^{1-u}}{\ln \left(r^{1-u}+\sqrt{\theta}\right)}\right) \\
& \geq \mathbb{P}(B(1) \geq 1) \times \frac{t}{2 r \ln r}, \quad \text { for small } t, \text { indep. of } u,
\end{aligned}
$$

arguing on the second line that $R$ dominates Brownian motion by comparing the drift. Combined with (3.10) and (3.11), this completes the proof of (ii). 


\subsection{Tail estimate for $U$}

Recall Hoeffding's inequality [13], or Th. 2.8 in [4]: for $b<1, c>1$ and $i \geq 1$,

$$
\mathbb{P}\left[2\left(U_{1}+\ldots+U_{i}\right) \geq c . i\right] \leq \exp \left(-\frac{i}{2}(c-1)^{2}\right)
$$

and

$$
\mathbb{P}\left[2\left(U_{1}+\ldots+U_{i}\right) \leq b . i\right] \leq \exp \left(-\frac{i}{2}(1-b)^{2}\right) .
$$

Remark 3.8 (The random difference equation (1.4)). Introduce the sequence

$$
S_{n}=\frac{T_{n}}{A_{n}^{2}}
$$

which is key in Section 6. In view of (3.1), we see that it solves the recursion

$$
S_{n+1}=\alpha_{n+1} S_{n}+\beta_{n+1}
$$

(i.e., (1.4) above), with

$$
\alpha_{n}=\left(A_{n}^{\prime}\right)^{-2}, \quad \beta_{n}=\frac{T_{n}^{\prime}}{\left(A_{n}^{\prime}\right)^{2}} .
$$

The bi-dimensional sequence $\left(\alpha_{n}, \beta_{n}\right), n \geq 1$, is i.i.d., and the sequence $\left(S_{n}\right)$ falls into the usual setup of random difference equation. In our case, the following quantities exist

$$
a:=\mathbb{E}\left[\ln \alpha_{1}\right], \quad b:=\lim _{t \rightarrow \infty} \mathbb{P}\left[\beta_{1}>t\right] \times \ln t,
$$

and satisfy $a<0$ (contractive case), $0<b<\infty$ (very heavy tail). Following [1] and [32], this prevents the Markov chain $S_{n}$ to be positive recurrent: though the contraction brings stability to the process, yet occasional large values of $\beta_{n}$ overcompensate this behavior so that positive recurrence fails to hold. In our case, we easily check from (3.3) that

$$
b=-a \quad(=\ln r),
$$

in which case the Markov chain $S_{n}$ is null recurrent, but in a critical manner: the chain is transient if $b>-a$ and null recurrent if $b \leq-a$.

\section{Proofs for section 2.2}

We consider the process $R$ from (1.1) on a geometric scale,

$$
X(t)=e^{-t / 2} R\left(e^{t}-1\right)
$$

and we observe that

$$
\beta(t)=\int_{0}^{e^{t}-1} \frac{1}{\sqrt{1+s}} d B(s)
$$

is a standard Brownian motion by Paul Lévy's characterization. We claim that $X$ solves the stochastic differential equation

$$
\left\{\begin{aligned}
d X(t) & =\left(\frac{1}{2 X(t)}-\frac{X(t)}{2}+\frac{1}{X(t) \ln \left[e^{t / 2} X(t)\right]}\right) d t+d \beta(t) \\
X(0) & =R(0) .
\end{aligned}\right.
$$

Indeed,

$$
\begin{aligned}
X(t) & =e^{-t / 2} X(0)+e^{-t / 2} \int_{0}^{e^{t}-1}\left(\frac{1}{2 R(s)}+\frac{1}{R(s) \ln R(s)}\right) d s+e^{-t / 2} B\left(e^{t}-1\right) \\
& =J(t)+K(t)+L(t)
\end{aligned}
$$


with $d J(t)=-\frac{1}{2} J(t) d t$, and

$$
\begin{aligned}
\frac{d K(t)}{d t} & =-\frac{1}{2} K(t)+\frac{1}{2 X(t)}+\frac{1}{X(t) \ln \left[e^{t / 2} X(t)\right]}, \\
d L(t) & =-\frac{1}{2} L(t) d t+e^{-t / 2} d B\left(e^{t}-1\right) .
\end{aligned}
$$

Moreover, we easily check the equality

$$
\int_{0}^{t} e^{-s / 2} d B\left(e^{s}-1\right)=\int_{0}^{e^{t}-1} \frac{1}{\sqrt{1+u}} d B(u)
$$

in the Gaussian space generated by $B$. Adding up terms, we see that $X$ solves the stochastic differential equation (4.2). Denote by $b_{t}$, resp. $b_{\infty}$ the drift coefficient and its limit, given for $x \in(0, \infty)$ by

$$
b_{t}(x)=\frac{1}{2 x}-\frac{x}{2}+\frac{1}{x(\ln x+t / 2)}, \quad b_{\infty}(x)=\frac{1}{2 x}-\frac{x}{2},
$$

and by $X^{(\infty)}$ the homogeneous diffusion

$$
d X^{(\infty)}(t)=\left(\frac{1}{2 X^{(\infty)}(t)}-\frac{X^{(\infty)}(t)}{2}\right) d t+d \beta(t) .
$$

Following the approach of Takeyama [29], we state the following

Lemma 4.1. The diffusion $X(t)=e^{-t / 2} R\left(e^{t}-1\right)$ is asymptotically homogeneous with homogeneous limit $X^{(\infty)}$, i.e, for all continuous $f$ with compact support in $(0, \infty)$ and all $t>0$,

$$
E[f(X(t+s)) \mid X(s)=x] \longrightarrow E_{x}\left[f\left(X^{(\infty)}(t)\right)\right] \quad \text { as } s \rightarrow \infty
$$

uniformly on compact subsets of $(0, \infty)$.

Proof. It is easier to consider $\widehat{X}(t)=X(t)-e^{-t / 2}$ which takes values in the fixed interval $(0, \infty)$, and $\widehat{X^{(s)}}(t)=\widehat{X}(s+t)$. Then, the coefficients of the diffusion $\widehat{X^{(s)}}$ converge to those of $X^{(\infty)}$, uniformly on compact subsets of $(0, \infty)$, and the corresponding martingale problems have a unique solution. Thus, Theorem 11.1.4 in [25] yields the desired result.

The process $X^{(\infty)}$ is the transform $X^{(\infty)}(t)=X^{(\infty, 2)}(t)=e^{-t / 2} \operatorname{BES}^{2}\left(e^{t}-1\right)$ of $\operatorname{BES}^{2}$ by the rescaling and deterministic time-change (4.1). It is recurrent and ergodic on $(0, \infty)$ with the Rayleigh law as invariant probability measure,

$$
d \nu(x)=x e^{-x^{2} / 2} \mathbb{1}_{(0, \infty)}(x) d x .
$$

A first consequence is that $R$ marginally behaves like $\mathrm{BES}^{2}$.

Corollary 4.2 (Convergence in law). Let $Z \sim \nu$. As $t \rightarrow \infty$,

$$
\frac{R(t)}{\sqrt{t}} \stackrel{\text { law }}{\longrightarrow} Z \text {. }
$$

Proof. Denote by $P_{s, t}, P_{s, t}^{(\infty)}(0 \leq s \leq t)$ the Markov semi-groups associated to $X$ and $X^{(\infty)}$,

$$
\left(P_{s, t} f\right)(x)=E[f(X(t)) \mid X(s)=x], \quad\left(P_{s, t}^{(\infty)} f\right)(x)=E\left[f\left(X^{(\infty)}(t)\right) \mid X^{(\infty)}(s)=x\right],
$$


so that $P_{s, t}^{(\infty)}=P_{0, t-s}^{(\infty)}$. For a bounded continuous $f:(0, \infty) \rightarrow \mathbb{R}$ we write for $x \geq 1$

$$
\begin{aligned}
P_{0, t+s} f(x) & -\int f d \nu=P_{0, s}\left(P_{s, s+t} f\right)(x)-\int f d \nu \\
& =P_{0, s}\left(P_{s, s+t} f-P_{s, s+t}^{(\infty)} f\right)(x)+P_{0, s}\left(P_{s, s+t}^{(\infty)} f-\int f d \nu\right)(x),
\end{aligned}
$$

where both terms vanish as $s, t \rightarrow \infty$, which is our claim. Indeed, by convergence of $X^{(\infty)}$ to equilibrium, $P_{s, s+t}^{(\infty)} f-\int f d \nu=P_{0, t}^{(\infty)} f-\int f d \nu \rightarrow 0$ uniformly on compact subsets of $(0, \infty)$ as $t \rightarrow \infty$ and Lemma 4.1 implies that $P_{s, s+t} f-P_{s, s+t}^{(\infty)} f \rightarrow 0$ uniformly on compacts as $s \rightarrow \infty$ : thus, we only need to prove tightness, i.e. that for all $x \geq 1$,

$$
\inf \left\{P_{0, s}\left(\mathbb{1}_{[\varepsilon, 1 / \varepsilon]}\right)(x) ; s \geq 1\right\} \rightarrow 1 \quad \text { as } \varepsilon \rightarrow 0 .
$$

But this follows from the next two bounds

- $R \geq \mathrm{BES}^{2}$ (see Proposition 3.5 (i)) which implies that $X \geq X^{(\infty)}$,

- $\sup _{s \geq 1} E\left[X(s)^{2} \mid X(0)=x\right] \leq \sup _{s \geq 1} s^{-1} E_{x}\left[R(s)^{2}\right]<\infty$ that we explain now.

First recall from [9] that $\frac{1}{\ln R}$ is a local martingale. Since it is positive, by Fatou's lemma it is also a super-martingale when started at $r>1$ and thus,

$$
E_{r}\left[\frac{1}{\ln R(t)}\right] \leq \frac{1}{\ln r} .
$$

By Itô's formula,

$$
d\left(R^{2}\right)=2\left(1+\frac{1}{\ln R(t)}\right) d t+2 R(t) d B(t)
$$

Thus, for all $r>1$,

$$
E_{r}\left[R(t)^{2}\right] \leq r^{2}+2 t\left(1+\frac{1}{\ln r}\right)
$$

We now consider the process starting from $R(0)=1$. Integrating (4.4), we get

$$
\begin{aligned}
& E_{1}\left[\left(R(t)^{2}-r^{2}\right) \mathbb{1}_{\tau(r)<t}\right]=2 E_{1}\left[\int_{0}^{t} \mathbb{1}_{\tau(r)<s}\left(1+\frac{1}{\ln R(s)}\right) d s+\int_{0}^{t} \mathbb{1}_{\tau(r)<s} R(s) d B(s)\right] \\
& \stackrel{\text { Markov }}{=} 2 \int_{0}^{t} E_{1}\left[\mathbb{1}_{\tau(r)<s} E_{r}\left(1+\frac{1}{\ln R(\cdot)}\right)_{\cdot=s-\tau(r)}\right] d s+0 \\
& \leq 2\left(1+\frac{1}{\ln r}\right) E_{1}\left[(t-\tau(r))^{+}\right]
\end{aligned}
$$

by (4.3). Finally we obtain that

$$
E_{1} R(t)^{2}=E_{1}\left[R(t)^{2} \mathbb{1}_{\tau(r) \geq t}\right]+E_{1}\left[R(t)^{2} \mathbb{1}_{\tau(r)<t}\right] \leq 2 r^{2}+2 t\left(1+\frac{1}{\ln r}\right)
$$

for any $r>1$. The corollary is proved.

Remark 4.3 (The local martingale $\frac{1}{\ln R}$ is not a martingale). Indeed, in the opposite case we would have the equality in (4.3), and further, $E_{r}\left[R(t)^{2}\right]=r^{2}+2 t\left(1+\frac{1}{\ln r}\right)$ for all $r>1$. This would contradict monotonicity, namely that for all $t>1, E_{r}\left[R(t)^{2}\right]$ is non-decreasing in $r>1$. Observe that we can actually compute these quantities, using equation (2.7) in [9]. We get for $|x|=r>1: E_{r}\left[\frac{1}{\ln R(t)}\right]=\frac{P_{x}(\tau(W ; 1) \geq t)}{\ln r}$, which is smaller than $\frac{1}{\ln r}$ for $t>0$, and further, $E_{r}\left[R(t)^{2}\right]=r^{2}+2 t+\frac{2 E_{x}[\tau(W ; 1) \wedge t]}{\ln r}$. 
Corollary 4.4 (Pointwise ergodic theorem). For all bounded continuous $f$ on $(0, \infty)$, as $t \rightarrow \infty$,

$$
\frac{1}{t} \int_{0}^{t} f(X(s)) d s \longrightarrow \int_{\mathbb{R}} f d \nu \quad \text { a.s. },
$$

or, equivalently,

$$
\frac{1}{t} \int_{0}^{e^{t}-1} f\left(\frac{R(u)}{\sqrt{1+u}}\right) \frac{1}{1+u} d u \longrightarrow \int_{\mathbb{R}} f d \nu \quad \text { a.s. }
$$

Proof. It is easy to check that, w.l.o.g., we can assume that $f:(0, \infty) \rightarrow \mathbb{R}$ is nondecreasing. By the comparison principles of Proposition 3.5, we can couple the processes $R, \mathrm{BES}^{2}, \operatorname{BES}^{2+\delta}(\delta>0)$ starting at 1 such that, a.s., for all $t \geq \ln (1+\sigma)$ with

$$
\sigma=\sup \left\{s>0: R(s) \leq e^{2 / \delta}\right\}<\infty,
$$

we have

$$
X^{(\infty, 2)}(t) \leq X(t) \leq X^{(\infty, 2+\delta)}(t)-e^{-t / 2}\left(\operatorname{BES}^{2+\delta}(\sigma)-e^{2 / \delta}\right) .
$$

By the pointwise ergodic theorem for $X^{(\infty, 2)}$ and $X^{(\infty, 2+\delta)}$ and monotonicity of $f$, we derive

$$
\int f d \nu \leq \liminf _{t \rightarrow \infty} \frac{1}{t} \int_{0}^{t} f(X(s)) d s \leq \limsup _{t \rightarrow \infty} \frac{1}{t} \int_{0}^{t} f(X(s)) d s \leq \int f d \nu_{\delta},
$$

where $d \nu_{\delta}(x)=c_{\delta} x^{1+\delta / 2} e^{-x^{2} / 2} \mathbb{1}_{(0, \infty)}(x) d x$ is the invariant law of $X^{(\infty, 2+\delta)}$. As $\delta$ vanishes, the two extreme members coincide, ending the proof of the first statement. The second one follows by changing variables.

\section{Proof of Theorem 2.1}

Recall the representation (3.1) from Corollary 3.3,

$$
T_{k}=T_{1}^{\prime}+A_{1}^{\prime 2} T_{2}^{\prime}+\ldots+\left(A_{1}^{\prime} \ldots A_{k-1}^{\prime}\right)^{2} T_{k}^{\prime}, \quad A_{k}=A_{1}^{\prime} \ldots A_{k}^{\prime}
$$

with $\left(T_{k}^{\prime}, A_{k}^{\prime}\right)_{k \geq 1}$ an i.i.d. sequence with the same law as $\left(T_{1}, A_{1}\right)$.

Fix $r_{ \pm}$with $1<r_{-}<r<r^{+}<\infty$. By (3.12) and (3.13), with probability one there exists some finite random $k_{0}$ such that for all $k \geq k_{0}$

$$
r_{-}^{k / 2} \leq A_{1}^{\prime} \ldots A_{k}^{\prime}=r^{U_{1}+\ldots+U_{k}} \leq r_{+}^{k / 2} .
$$

In what follows we will use the rough bounds

$$
\max _{i=1, \ldots, k} T_{i}^{\prime} \leq T_{k} \leq T_{k_{0}}+\left(k-k_{0}\right) \max _{i=1, \ldots, k} r_{+}^{i-1} T_{i}^{\prime} .
$$

Lemma 5.1. There exists a constant $c$ such that for all sequence $(\delta(k))_{k}$ tending to 0 , we have

$$
\mathbb{P}\left[k \max _{i=1, \ldots, k} r_{+}^{i-1} T_{i}^{\prime} \geq e^{k / \delta(k)}\right] \leq c \delta(k)
$$

eventually.

Proof. Fix $a$ with $1<a<e$. Letting $v_{k}=a^{\frac{k}{\delta(k)}}$ and $t_{k}=k r_{+}^{k} v_{k}$, we note that $e^{\frac{k}{\delta(k)}} \geq t_{k}$ eventually since $\delta$ vanishes, and we have by independence

$$
\mathbb{P}\left[k \max _{i=1, \ldots, k} r_{+}^{i-1} T_{i}^{\prime}<t_{k}\right]=\Pi_{i=1}^{k} \mathbb{P}\left[T_{i}^{\prime}<r_{+}^{k-i+1} v_{k}\right]
$$


From Proposition 3.6 there exists $c_{1}>0$ such that for all $t>1$

$$
\mathbb{P}\left(T_{1} \geq t\right) \leq \frac{c_{1}}{\ln t}
$$

and since $v_{k} \rightarrow \infty$ as $k \rightarrow \infty$, we have for all large enough $k$,

$$
\begin{aligned}
\mathbb{P}\left[k \max _{i=1, \ldots, k} r_{+}^{i-1} T_{i}^{\prime}<t_{k}\right] & \geq \Pi_{i=1}^{k}\left(1-\frac{c_{1}}{\ln \left(r_{+}^{k-i+1} v_{k}\right)}\right) \\
& =\Pi_{i=1}^{k}\left(1-\frac{c_{1}}{\ln \left(r_{+}^{i} v_{k}\right)}\right) \\
& \geq \exp \left(-2 c_{1} \sum_{i=1}^{k} \frac{1}{i \ln r_{+}+\ln v_{k}}\right) \\
& \geq \exp \left(-\frac{2 c_{1}}{\ln r_{+}} \ln \left(\frac{k \ln r_{+}+\ln v_{k}}{\ln v_{k}}\right)\right) \\
& =\exp \left(-\frac{2 c_{1}}{\ln r_{+}} \ln \left(1+\frac{\ln r_{+}}{\ln a} \delta(k)\right)\right) \\
& \geq 1-c \delta(k)
\end{aligned}
$$

with $c=2 c_{1} / \ln a$ for all large $k$, since $\delta$ vanishes at $\infty$. This ends the proof.

Proof. Theorem 2.1, claim (2.3). Let

$$
\delta(t)=g(\ln t), \quad \kappa(i)=2^{i}, i \geq 1, \quad \mathcal{K}=\{\kappa(i): i \geq 1\} .
$$

Define, for $x \geq 2,\lfloor x\rfloor_{\mathcal{K}}=\max \{k \in \mathcal{K}: k \leq x\}=2^{\lfloor(\ln x) /(\ln 2)\rfloor}$. Note that

$$
x \geq\lfloor x\rfloor_{\mathcal{K}} \geq x / 2
$$

First, since $g$ is non-increasing,

$$
\begin{aligned}
\sum_{k \in \mathcal{K}} \delta(k) & =\sum_{i \geq 1} \delta(k(i)) \\
& =\sum_{i \geq 1} g(\ln k(i)) \\
& =\sum_{i \geq 1} g(i \ln 2) \\
& \leq \frac{1}{\ln 2} \sum_{i \geq 1} \int_{(i-1) \ln 2}^{i \ln 2} g(t) d t \\
& =\frac{1}{\ln 2} \int_{0}^{\infty} g(t) d t<\infty
\end{aligned}
$$

Fix a constant $c_{2}>0$ to be chosen later and $c_{3}=c_{2}^{-1}$. Combining Borel-Cantelli's lemma and Lemma 5.1, we have a.s.

$$
k \max _{i=1, \ldots, k} r_{+}^{i-1} T_{i}^{\prime}<e^{c_{2} k / \delta(k)} \quad \text { for all } k \in \mathcal{K} \text { large enough }
$$

and, in addition to (5.1), we have for large $k \in \mathcal{K}$,

$$
T_{k} \leq T_{k_{0}}+\frac{k-k_{0}}{k} e^{c_{2} k / \delta(k)} \leq e^{c_{2} k / \delta(k)}
$$


since $g$ is non-increasing. By integrability, $g$ is vanishing at infinity, so the function

$$
f(t)=c_{3}(\ln t) g\left(\ln _{2} t\right)
$$

is such that $f(t) \leq \ln t$ eventually, and also $g\left(\ln _{2} t\right) \leq g(\ln f(t))$ by monotonicity. Thus, for large $k$ and $t$ 's,

$$
\begin{aligned}
k \leq c_{3}(\ln t) \delta(\ln t) & =f(t) \quad \text { implies that } \\
\frac{k}{\delta(k)} & =\frac{k}{g(\ln k)} \leq \frac{f(t)}{g(\ln f(t))}=\frac{c_{3}(\ln t) g\left(\ln _{2} t\right)}{g(\ln f(t))} \leq c_{3} \ln t .
\end{aligned}
$$

Now, define random integers $k(t)=\max \left\{k \in \mathcal{K} ; T_{k} \leq t\right\}$, and note from (5.4) that a.s., for large $t$ we have $k(t) \geq \max \left\{k \in \mathcal{K} ; e^{c_{2} \frac{k}{\delta(k)}} \leq t\right\}$. Then, a.s., for all large enough $t$,

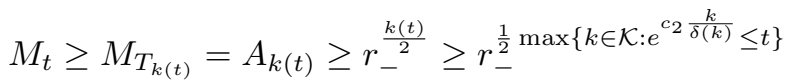

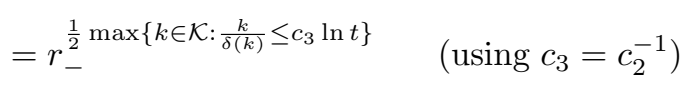

$$
\begin{aligned}
& \geq r_{-}^{\frac{1}{2}} \max \{k \in \mathcal{K}: k \leq f(t)\} \\
& =r_{-}^{\frac{1}{2}\left\lfloor c_{3}(\ln t) \delta(\ln t)\right\rfloor_{\mathcal{K}}} \\
& \geq r_{-}^{\frac{c_{3}}{4}(\ln t) \delta(\ln t)}
\end{aligned}
$$

Taking $c_{3}=c_{2}^{-1}>4 / \ln r_{-}$, we conclude that a.s., $M(t) \geq e^{(\ln t) g\left(\ln _{2} t\right)}$ eventually, ending the proof of (2.3).

We now turn to the proof of claim (2.4) of Theorem 2.1. We start with a lemma:

Lemma 5.2. Let $\left(n_{k}\right)_{k \geq 0}$ be a non-decreasing sequence of integers and $\left(t_{k}\right)_{k \geq 0}$ be a sequence with $t_{k}>1$. Then,

$$
\sum_{k \geq 0} \frac{n_{k+1}-n_{k}}{\ln t_{k+1}}=\infty \quad \Longrightarrow \quad \text { a.s., } T_{n_{k}} \geq t_{k} \text { infinitely often. }
$$

Proof. The events $E_{k}=\left\{\max _{i=n_{k}+1, \ldots, n_{k+1}} T_{i}^{\prime} \geq t_{k+1}\right\}, k \geq 0$ are independent with $E_{k} \subset\left\{T_{n_{k+1}} \geq t_{k+1}\right\}$. Hence the conclusion holds as soon as these events occurs infinitely often a.s. By the second Borel-Cantelli lemma, it suffices to show that the assumption implies $\sum_{k \geq 0} \mathrm{P}\left(E_{k}\right)=\infty$. We use Proposition 3.6 and independence. The case when $t_{k}$ does not tend to infinity is easily considered, so we assume from now on that $k$ is large enough so that $\mathbb{P}\left(T \geq t_{k+1}\right) \geq c / \ln t_{k+1}$ for some fixed constant $c \in(0, \ln r)$. Then, we can bound

$$
\begin{aligned}
\mathbb{P}\left(E_{k}\right) & =1-\mathbb{P}\left(T \leq t_{k+1}\right)^{n_{k+1}-n_{k}} \\
& \geq 1-\left(1-\frac{c}{\ln t_{k+1}}\right)^{n_{k+1}-n_{k}} \\
& \geq 1-\exp \left(-\frac{c\left(n_{k+1}-n_{k}\right)}{\ln t_{k+1}}\right)
\end{aligned}
$$

which is the general term of a divergent series.

Proof. Theorem 2.1, claim (2.4). Let us consider

$$
t_{k}=e^{e^{k}}, \quad n_{k}=\left\lfloor f\left(t_{k}\right)\right\rfloor, \quad f(t)=c_{3}(\ln t) g\left(\ln _{2} t\right)
$$


with $c_{3}>0$ to be fixed later. Note that $f$ is non-decreasing by assumption. We have

$$
\begin{aligned}
\sum_{k \geq 0} \frac{n_{k+1}-n_{k}}{\ln t_{k+1}}=\sum_{k \geq 0} \frac{\left\lfloor f\left(t_{k+1}\right)\right\rfloor-\left\lfloor f\left(t_{k}\right)\right\rfloor}{\ln t_{k+1}} & =\sum_{k \geq 0} \frac{f\left(t_{k+1}\right)-f\left(t_{k}\right)}{\ln t_{k+1}}+c_{4} \\
& =c_{3} \sum_{k \geq 0} g(k+1)-\frac{1}{e} g(k)+c_{4}
\end{aligned}
$$

with a constant $c_{4}$ which is finite since $t_{k}$ is increasing fast and the truncation error is bounded. As in (5.3), $\sum_{k \geq 0} g(k) \geq \int_{0}^{\infty} g(t) d t=\infty$, and

$$
\sum_{k=0}^{n} g(k+1)-\frac{1}{e} g(k)=g(n+1)-\frac{1}{e} g(0)+\left(1-\frac{1}{e}\right) \sum_{k=1}^{n} g(k) .
$$

Therefore $\sum_{k \geq 0} \frac{n_{k+1}-n_{k}}{\ln t_{k+1}}=\infty$. From Lemma 5.2 we obtain that a.s., $T_{n_{k}} \geq t_{k}$ i.o., which shows that

$$
M_{t_{k}} \leq M_{T_{n_{k}}}=A_{n_{k}} \leq r_{+}^{n_{k}} \leq r_{+}^{f\left(t_{k}\right)}
$$

Taking $c_{3}<1 / \ln r_{+}$, we obtain the desired claim.

\section{Proof of Theorem 2.2}

We study the sequence

$$
S_{n}=\frac{T_{n}}{A_{n}^{2}}=\sum_{i=1}^{n} \frac{T_{i}^{\prime} A_{i-1}^{2}}{A_{n}^{2}}=\sum_{i=1}^{n} \frac{T_{i}^{\prime}}{r^{2\left(U_{i}+\cdots+U_{n}\right)}}
$$

which can be written in the form

$$
S_{m}=\frac{S_{n}}{r^{2\left(U_{n+1}+\cdots+U_{m}\right)}}+S_{n+1}^{m}
$$

where, for $1 \leq n<m$,

$$
S_{n+1}^{m}=\sum_{i=n+1}^{m} \frac{T_{i}^{\prime}}{r^{2\left(U_{i}+\cdots+U_{m}\right)}}
$$

The point is that, in (6.1), $S_{n}$ and $S_{n+1}^{m}$ are independent, with $S_{n+1}^{m}$ equal to $S_{m-n}$ in law.

We study the convergence/divergence of the series $\sum_{n \geq 1} \mathbb{P}\left[S_{n} \leq t_{n}\right]$, with $t_{n}$ of the form

$$
t_{n}=\frac{\beta}{\ln _{2} n} \wedge 1
$$

for some $\beta>0$.

\subsection{Proof of (2.5)}

Let $\left(i^{(n)}\right)_{n \geq 1}$ be a sequence of integers such that $1 \leq i^{(n)} \leq n$ and $\left(c_{i}^{(n)}\right)_{i=i^{(n)}+1, \ldots, n, n \geq 1}$ be a doubly-indexed sequence of real parameters with $c_{i}^{(n)}>1$, to be fixed later on. 


\section{Upper bound:}

From (6.1) we have

$$
\begin{aligned}
& \mathbb{P}\left[S_{n} \leq t_{n}\right] \leq \mathbb{P}\left[\frac{T_{1}^{\prime}}{r^{2\left(U_{1}+\cdots+U_{n}\right)}} \leq t_{n}, S_{2}^{n} \leq t_{n}\right] \\
& \leq \mathbb{P}\left[\frac{T_{1}^{\prime}}{r^{2\left(U_{1}+\cdots+U_{n}\right)}} \leq t_{n}, S_{2}^{n} \leq t_{n}, 2\left(U_{1}+\cdots+U_{n}\right) \leq c_{n}^{(n)} \cdot n\right] \\
& +\mathbb{P}\left[2\left(U_{1}+\cdots+U_{n}\right)>c_{n}^{(n)} \cdot n\right] \\
& \leq \mathbb{P}\left[T_{1}^{\prime} \leq t_{n} r_{n}^{c_{n}^{(n)} \cdot n}, S_{2}^{n} \leq t_{n}\right]+\mathbb{P}\left[2\left(U_{1}+\cdots+U_{n}\right)>c_{n}^{(n)} \cdot n\right] \\
& \leq \mathbb{P}\left[T \leq t_{n} r^{c_{n}^{(n)} \cdot n}\right] \times \mathbb{P}\left[S_{n-1} \leq t_{n}\right]+\mathbb{P}\left[2\left(U_{1}+\cdots+U_{n}\right)>c_{n}^{(n)} . n\right] \text {. }
\end{aligned}
$$

Iterating the estimate,

$$
\mathbb{P}\left[S_{n-1} \leq t_{n}\right] \leq \mathbb{P}\left[T \leq t_{n} r^{c_{n-1}^{(n)} \cdot(n-1)}\right] \times \mathbb{P}\left[S_{n-2} \leq t_{n}\right]+\mathbb{P}\left[2\left(U_{1}+\cdots+U_{n-1}\right)>c_{n-1}^{(n)} \cdot(n-1)\right],
$$

and so on down to $i^{(n)}+1$, we obtain

$$
\begin{aligned}
\mathbb{P}\left[S_{n} \leq t_{n}\right] \leq & \left(\prod_{i=i^{(n)}+1}^{n} \mathbb{P}\left[T \leq t_{n} r^{c_{i}^{(n)} \cdot i}\right]\right) \times \mathbb{P}\left[S_{i(n)} \leq t_{n}\right] \\
& +\sum_{i=i^{(n)}+1}^{n}\left(\prod_{j=i+1}^{n} \mathbb{P}\left[T \leq t_{n} r^{c_{j}^{(n)} \cdot j}\right]\right) \times \mathbb{P}\left[2\left(U_{1}+\ldots+U_{i}\right)>c_{i}^{(n)} \cdot i\right] .
\end{aligned}
$$

\section{Choice of $i^{(n)}$ and the $c_{i}^{(n)}$}

Let $i^{(n)}=\left\lfloor\ln _{2} n\right\rfloor$ and for $i^{(n)}+1 \leq i \leq n$,

$$
c_{i}^{(n)}=1+\sqrt{\frac{8}{i}\left(\ln i+\ln _{2} n\right)} .
$$

We have for $i^{(n)}+1 \leq i \leq n$ and large $n$,

$$
\begin{array}{rlr}
\ln \mathbb{P}\left[T \leq t_{n} r^{c_{i}^{(n)} . i}\right] & \leq \ln \mathbb{P}\left[T \leq r^{c_{i}^{(n)} .}\right] & \\
& \leq-\mathbb{P}\left[T \geq r^{c_{i}^{(n)} \cdot i}\right] & \\
& \leq-\frac{1}{c_{i}^{(n)} \cdot i}+\varepsilon_{n, i, 1} & (b y(6.2)) \\
& \leq-\frac{1}{i}+\varepsilon_{n, i, 2} & (\text { by }(6.4)),
\end{array}
$$

with error terms

$$
\varepsilon_{n, i, 1}=\frac{\ln _{2}\left(c_{i}^{(n)} \cdot i \ln r\right)+C}{\left(c_{i}^{(n)} \cdot i\right)^{2} \ln r}, \quad \varepsilon_{n, i, 2}=\varepsilon_{n, i, 1}+\sqrt{\frac{8}{i^{3}}\left(\ln i+\ln _{2} n\right)} .
$$

One can check that $\sup _{n} \sum_{i=i^{(n)}+1}^{n} \varepsilon_{n, i, 2}<\infty$, so for some positive constant $D$, for $n$ large and $i^{(n)} \leq i \leq n$,

$$
\begin{aligned}
\prod_{j=i+1}^{n} \mathbb{P}\left[T \leq t_{n} r^{c_{j}^{(n)} \cdot j}\right] & \leq \exp \left(-\sum_{j=i+1}^{n} \frac{1}{j}+\sum_{j=i+1}^{n} \varepsilon_{n, j, 2}\right) \\
& \leq D \exp \left(-\ln \left(\frac{n}{i}\right)\right)=D \frac{i}{n} .
\end{aligned}
$$


Combining this with (3.12), we get for $n$ large and $i^{(n)}+1 \leq i \leq n$,

$$
\begin{aligned}
\left(\prod_{j=i+1}^{n} \mathbb{P}\left[T \leq t_{n} r^{c_{j}^{(n)} \cdot j}\right]\right) \times \mathbb{P}\left[2\left(U_{1}+\ldots+U_{i}\right)>c_{i}^{(n)} \cdot i\right] & \leq D \frac{i}{n} \exp \left(-4\left(\ln i+\ln _{2} n\right)\right) \\
& =\frac{D}{i^{3} n(\ln n)^{4}} .
\end{aligned}
$$

Thus, the series $\sum a_{n}$, with

$$
a_{n}=\sum_{i=i^{(n)}+1}^{n}\left(\prod_{j=i+1}^{n} \mathbb{P}\left[T \leq t_{n} r^{c_{j}^{(n)} \cdot j}\right]\right) \times \mathbb{P}\left[2\left(U_{1}+\ldots+U_{i}\right)>c_{i}^{(n)} . i\right]
$$

is convergent.

\section{Choice of $t_{n}$}

To conclude, we need to take care of the first term in the right-hand side of (6.3). Recall $t_{n}$ from (6.2) (we will assume $n$ large so that $\ln _{2} n \geq \beta$ ), and fix an integer $i_{1} \geq 1$ and an $\epsilon \in(0, r-1)$. For $1 \leq i \leq i_{1}$, applying (3.7) we get as $n \rightarrow \infty$,

$$
\mathbb{P}\left[T \leq t_{n} r^{2 i}\right] \leq \exp \left(-\frac{(r-1-\epsilon)^{2}}{2 \beta r^{2 i}} \ln _{2} n\right)
$$

and then, for $n$ large,

$$
\begin{aligned}
\mathbb{P}\left[S_{i(n)} \leq t_{n}\right] & \leq \mathbb{P}\left[T_{i}^{\prime} \leq t_{n} r^{2 i}, i=1, \ldots, i_{1}\right] \\
& =\prod_{i=1}^{i_{1}} \mathbb{P}\left[T \leq t_{n} r^{2 i}\right] \\
& \leq \exp \left(-\sum_{i=1}^{i_{1}} \frac{(r-1-\epsilon)^{2}}{2 \beta r^{2 i}} \ln _{2} n\right) \\
& \leq \exp \left(-\frac{(r-1-\epsilon)^{2}}{2 \beta} \frac{1}{r^{2}} \frac{1-\left(\frac{1}{r^{2}}\right)^{i_{1}}}{1-\frac{1}{r^{2}}} \ln _{2} n\right) \\
& \leq(\ln n)^{-\frac{(r-1-\epsilon)^{2}}{2 \beta\left(r^{2}-1\right)}\left(1-\left(\frac{1}{r^{2}}\right)^{i_{1}}\right) .}
\end{aligned}
$$

Using (6.5) we will bound

$$
\left(\prod_{i=i^{(n)}+1}^{n} \mathbb{P}\left[T \leq t_{n} r^{c_{i}^{(n)} \cdot i}\right]\right) \times \mathbb{P}\left[S_{i(n)} \leq t_{n}\right] \leq D \frac{i^{(n)}}{n}(\ln n)^{-\frac{(r-1-\epsilon)^{2}}{2 \beta\left(r^{2}-1\right)}\left(1-\left(\frac{1}{r^{2}}\right)^{i_{1}}\right)}
$$

where $i^{(n)}=\left\lfloor\ln _{2} n\right\rfloor$. As soon as $\beta<\frac{(r-1)}{2(r+1)}$, there exists some integer $i_{1}$ and some $\epsilon \in(0, r-1)$ such that

$$
\frac{(r-1-\epsilon)^{2}}{2 \beta\left(r^{2}-1\right)}\left(1-\left(\frac{1}{r^{2}}\right)^{i_{1}}\right)>1
$$

and combining (6.3) with $\sum_{n} a_{n}<\infty$, we obtain $\sum \mathbb{P}\left(S_{n} \leq t_{n}\right)<\infty$, i.e.,

$$
\sum_{n \geq 1} \mathbb{P}\left[T_{n} \leq A_{n}^{2} t_{n}\right]<\infty
$$




\section{Conclusion}

Let $\beta<\frac{(r-1)}{2(r+1)}$. It follows from Borel-Cantelli's lemma that a.s., eventually

$$
T_{n} \geq \frac{\beta A_{n}^{2}}{\ln _{2} n}
$$

Now, for $T_{n} \leq t \leq T_{n+1}$, if $n$ is large enough,

$$
M_{t} \leq M_{T_{n+1}}=A_{n+1} \leq r A_{n} \leq r \sqrt{\beta^{-1} T_{n} \ln _{2} n} \leq r \sqrt{\beta^{-1} t \ln _{2} n},
$$

and since we have $T_{n} \geq \frac{\beta A_{n}^{2}}{\ln _{2} n} \geq r_{-}^{\frac{n}{2}}$ for $n$ large enough, we have $t \geq r_{-}^{\frac{n}{2}}$, and $n \leq \frac{2 \ln t}{\ln r_{-}}$. Finally,

$$
M_{t} \leq r \sqrt{\beta^{-1} t \ln _{2}\left(\frac{2 \ln t}{\ln r_{-}}\right)}
$$

Hence, we have proved (2.5) with any $K>r \sqrt{\frac{2(r+1)}{(r-1)}}$.

\subsection{Proof of (2.6)}

We start by proving that it suffices to show divergence of the series introduced above (6.2):

Lemma 6.1. Let $\beta_{0}=\inf \left\{\beta>0: \sum_{n} \mathbb{P}\left(S_{n} \leq \frac{\beta}{\ln _{2} n}\right)=\infty\right\}$. Then

$$
\liminf _{n} S_{n} \ln _{2} n=\beta_{0} \quad \text { a.s. }
$$

Proof. For all $\beta<\beta_{0}$, we have $\sum_{n} \mathbb{P}\left(S_{n} \leq \frac{\beta}{\ln _{2} n}\right)<\infty$ and the first Borel-Cantelli lemma shows that $\liminf { }_{n} S_{n} \ln _{2} n \geq \beta_{0}$. To prove the reverse inequality we proceed by steps:

- First step: For any non-increasing sequence $\left(t_{n}\right)_{n}$,

$$
\sum_{n \geq 1} \mathbb{P}\left[S_{n} \leq t_{n}\right]=\infty \Longrightarrow \mathbb{P}\left(S_{n} \leq t_{n} \text { i.o. }\right) \geq \frac{1}{4} .
$$

Indeed, for $1 \leq n \leq m$,

$$
\begin{aligned}
\mathbb{P}\left[S_{n} \leq t_{n}, S_{m} \leq t_{m}\right] & \leq \mathbb{P}\left[S_{n} \leq t_{n}, S_{n+1}^{m} \leq t_{m}\right] \\
& =\mathbb{P}\left[S_{n} \leq t_{n}\right] \times \mathbb{P}\left[S_{n+1}^{m} \leq t_{m}\right] \\
& =\mathbb{P}\left[S_{n} \leq t_{n}\right] \times \mathbb{P}\left[S_{m-n} \leq t_{m}\right] \\
& \leq \mathbb{P}\left[S_{n} \leq t_{n}\right] \times \mathbb{P}\left[S_{m-n} \leq t_{m-n}\right]
\end{aligned}
$$

since $t_{m} \leq t_{m-n}$. Now, for $k \geq 1$,

$$
\begin{aligned}
\sum_{1 \leq n<m \leq k} \mathbb{P}\left[S_{n} \leq t_{n}, S_{m} \leq t_{m}\right] & \leq \sum_{1 \leq n<m \leq k} \mathbb{P}\left[S_{n} \leq t_{n}\right] \times \mathbb{P}\left[S_{m-n} \leq t_{m-n}\right] \\
& \leq \sum_{1 \leq n, m \leq k} \mathbb{P}\left[S_{n} \leq t_{n}\right] \times \mathbb{P}\left[S_{m} \leq t_{m}\right]
\end{aligned}
$$

For all $k$ large enough we have $\sum_{n=1}^{k} \mathbb{P}\left[S_{n} \leq t_{n}\right] \geq 2$, and then for all $1 \leq n \leq k$,

$$
\sum_{1 \leq m \leq k, m \neq n} \mathbb{P}\left[S_{m} \leq t_{m}\right] \geq 2-\mathbb{P}\left[S_{n} \leq t_{n}\right] \geq \mathbb{P}\left[S_{n} \leq t_{n}\right]
$$


Therefore,

$$
\begin{aligned}
\sum_{1 \leq n, m \leq k} \mathbb{P}\left[S_{n} \leq t_{n}\right] \times \mathbb{P}\left[S_{m} \leq t_{m}\right] & \leq 2 \sum_{1 \leq n, m \leq k, n \neq m} \mathbb{P}\left[S_{n} \leq t_{n}\right] \times \mathbb{P}\left[S_{m} \leq t_{m}\right] \\
& =4 \sum_{1 \leq n<m \leq k} \mathbb{P}\left[S_{n} \leq t_{n}\right] \times \mathbb{P}\left[S_{m} \leq t_{m}\right]
\end{aligned}
$$

Kochen-Stone's theorem [18] - a variant of Borel-Cantelli's lemma - yields

$$
\mathbb{P}\left[S_{n} \leq t_{n} \text { i.o. }\right] \geq \limsup _{k \geq 1} \frac{\sum_{1 \leq n<m \leq k} \mathbb{P}\left[S_{n} \leq t_{n}\right] \times \mathbb{P}\left[S_{m} \leq t_{m}\right]}{\sum_{1 \leq n<m \leq k} \mathbb{P}\left[S_{n} \leq t_{n}, S_{m} \leq t_{m}\right]} \geq \frac{1}{4},
$$

which concludes this step.

- Second step: Let's introduce the $\sigma$-fields

$$
\mathcal{A}_{k}=\sigma\left(\left(A_{n}^{\prime}, T_{n}^{\prime}\right) ; n \geq k\right), k=1,2 \ldots, \quad \mathcal{T}=\bigcap_{k \geq 1} \mathcal{A}_{k} .
$$

By Kolmogorov 0-1 law and independence of the sequence $\left(\left(A_{n}^{\prime}, T_{n}^{\prime}\right) ; n \geq 1\right)$, every element $A$ of the tail field $\mathcal{T}$ has $\mathbb{P}(A) \in\{0,1\}$. Fix $\beta \geq 0$ and introduce the events

$$
E=\left\{\liminf _{n} S_{n} \ln _{2} n \leq \beta\right\}, \quad E_{k}=\left\{\liminf _{n} S_{k+1}^{n+k} \ln _{2} n \leq \beta\right\},
$$

and

$$
\Omega_{0}=\left\{\lim _{n \rightarrow \infty} \frac{\ln _{2} n}{r^{2\left(U_{1}+\ldots+U_{n}\right)}}=0\right\} .
$$

Note that $E=E_{0}$ and that $\mathbb{P}\left(\Omega_{0}\right)=1$. Since, by definition,

$$
S_{k+1}^{n+k+1}=\frac{T_{k+1}^{\prime}}{r^{2\left(U_{k+1}+\ldots+U_{n+k+1}\right)}}+S_{k+2}^{n+k+1},
$$

we see that the two sets $E_{k}$ and $E_{k+1}$ coincide on $\Omega_{0}$, for all $k \geq 0$. Denoting the common intersection by

$$
\widehat{E}=E \cap \Omega_{0}=E_{k} \cap \Omega_{0},
$$

we see that $\widehat{E}$ belongs to $\mathcal{T}$ and then has probability equal to 0 or 1 . The similar $0-1$ law holds for $E$ which is equal to $\widehat{E}$ up to a negligible set.

- Final step: For any $\beta>\beta_{0}$, the series $\sum_{n} \mathbb{P}\left(S_{n} \leq t_{n}\right)$ with $t_{n}=\beta / \ln _{2} n$ is diverging. By the first step, the probability $\mathbb{P}\left[S_{n} \leq t_{n}\right.$ i.o. $] \geq 1 / 4$, and by the second one is equal to 1. Thus $\liminf { }_{n} S_{n} \ln _{2} n \leq \beta$ a.s., for all such $\beta$ 's. The lemma is proved.

Remark 6.2. We have followed the approach of the renewal structure to get the 0-1 law, with the advantage to keep the paper self-contained. A tempting alternative would be to show that the tail $\sigma$-field of $R$ is trivial; we mention the illuminating survey [23] on the tail $\sigma$-field of a diffusion.

Anticipating on the proof of (2.6) we now give a short proof of Theorem 1.2.

Proof. It is not difficult to check the criteria of [10] or [24] for triviality of the tail $\sigma$-field of one-dimensional diffusion (see Theorem 3 in [23]). Then, $K^{*}=\lim \sup _{t \rightarrow \infty} \frac{M(t)}{\sqrt{t \ln _{3} t}}$ is a.s. constant, and results (2.5) and (2.6) show that $K^{*}$ is positive and finite.

To continue the proof of (2.6) we need an intermediate result.

Lemma 6.3. For all $\alpha_{0}>0$, there exists $\beta>0$ such that, for all $n$ large enough,

$$
\mathbb{P}\left[S_{\left\lfloor\alpha_{0} \ln _{2} n\right\rfloor} \leq \frac{\beta}{\ln _{2} n}\right] \geq \frac{1}{\ln n} .
$$


Proof. Clearly, it suffices to prove that for $v>0$, there exists $u>0$ such that, for all large $n$, we have

$$
\mathbb{P}\left[S_{n} \leq \frac{u}{n}\right] \geq \frac{1}{e^{v n}} .
$$

Indeed, substituting $v, n$ in (6.6) by $\alpha_{0}^{-1},\left\lfloor\alpha_{0} \ln _{2} n\right\rfloor$ shows that any $\beta>u / \alpha_{0}$ fulfills the statement of the lemma.

To show (6.6), we fix some $b \in(0,1)$ ( $b$ will be chosen small later on), and we note that:

$$
U_{i} \geq b \text { and } T_{i}^{\prime} \leq \frac{u}{n}\left(r^{b}-1\right) r^{b(n-i+1)} \text { for all } i=1, \ldots, n
$$

imply that

$$
S_{n}=\sum_{i=1}^{n} \frac{T_{i}^{\prime}}{r^{2\left(U_{i}+\cdots+U_{n}\right)}} \leq \sum_{i=1}^{n} \frac{\frac{u}{n}\left(r^{b}-1\right) r^{b(n-i+1)}}{r^{2 b(n-i+1)}} \leq \frac{u}{n}
$$

Then,

$$
\begin{aligned}
\mathbb{P}\left[S_{n} \leq \frac{u}{n}\right] & \geq \prod_{i=1}^{n} \mathbb{P}\left[U_{i} \geq b, T_{i}^{\prime} \leq \frac{u}{n}\left(r^{b}-1\right) r^{b(n-i+1)}\right] \\
& =(1-b)^{n} \prod_{i=1}^{n} \mathbb{P}\left[T_{i}^{\prime} \leq \frac{u}{n}\left(r^{b}-1\right) r^{b(n-i+1)} \mid U_{i} \geq b\right] \\
& =(1-b)^{n} \prod_{i=1}^{n} \mathbb{P}\left[T \leq \frac{u}{n}\left(r^{b}-1\right) r^{b i} \mid U \geq b\right] .
\end{aligned}
$$

By Proposition 3.7, we can find $t_{0}>0$ and $\rho>0$ such that, for $t \leq t_{0}$,

$$
\mathbb{P}[T \leq t \mid U \geq b] \geq \exp \left(-\frac{\rho}{t}\right) .
$$

Now, we fix some $t_{1}>t_{0}$, we will bound the factors in (6.7) as follows:

For $\frac{\ln \left(t_{1} \frac{n}{u\left(r^{b}-1\right)}\right)}{b \ln r} \leq i \leq n$ :

$$
\mathbb{P}\left[T \leq \frac{u}{n}\left(r^{b}-1\right) r^{b i} \mid U \geq b\right] \geq \mathbb{P}\left[T \leq t_{1} \mid U \geq b\right],
$$

for $\frac{\ln \left(t_{0} \frac{n}{u\left(r^{b}-1\right)}\right)}{b \ln r} \leq i \leq \frac{\ln \left(t_{1} \frac{n}{u\left(r^{b}-1\right)}\right)}{b \ln r}$ :

$$
\mathbb{P}\left[T \leq \frac{u}{n}\left(r^{b}-1\right) r^{b i} \mid U \geq b\right] \geq \mathbb{P}\left[T \leq t_{0} \mid U \geq b\right],
$$

and for $1 \leq i \leq \frac{\ln \left(t_{0} \frac{n}{u\left(r^{b}-1\right)}\right)}{b \ln r}$ :

$$
\mathbb{P}\left[T \leq \frac{u}{n}\left(r^{b}-1\right) r^{b i} \mid U \geq b\right] \geq \exp \left(-\rho \frac{n}{u\left(r^{b}-1\right)} \frac{1}{r^{b i}}\right) .
$$

With this choice, the estimate (6.7) becomes

$$
\begin{aligned}
\mathbb{P}\left[S_{n} \leq \frac{u}{n}\right] & \geq(1-b)^{n} \times \mathbb{P}\left[T \leq t_{1} \mid U \geq b\right]^{n} \times \mathbb{P}\left[T \leq t_{0} \mid U \geq b\right]^{\frac{\ln \left(\frac{t_{1}}{t_{0}}\right)}{\ln r}+1} \\
& \times \prod_{i=1}^{\left\lfloor\frac{\ln \left(t_{0} \frac{n}{u\left(r^{b}-1\right)}\right)}{\prod_{\ln r}}\right\rfloor} \exp \left(-\rho \frac{n}{u\left(r^{b}-1\right)} \frac{1}{r^{b i}}\right) \\
& \geq(1-b)^{n} \times \mathbb{P}\left[T \leq t_{1} \mid U \geq b\right]^{n} \times \mathbb{P}\left[T \leq t_{0} \mid U \geq b\right]^{\frac{\ln \left(\frac{t_{1}}{t_{0}}\right)}{b \ln r}+1} \\
& \times \exp \left(-\rho \frac{n}{u\left(r^{b}-1\right)^{2}}\right) .
\end{aligned}
$$


From this we derive the claim (6.6) by taking $b$ small, $u$ and $t_{1}$ large. This ends the proof of the lemma.

Proof. Theorem 2.2, claim (2.6). Similarly to the proof of (2.5), we let $t_{n}=\frac{\beta}{\ln _{2} n} \wedge$ $1,\left(i^{(n)}\right)_{n \geq 1}$ be a sequence of integers, and $\left(b_{i}^{(n)}\right)_{i=i^{(n)}+1, \ldots n, n \geq 1}$ be a doubly-indexed sequence with $0<b_{i}^{(n)}<1$, given by

$$
b_{i}^{(n)}=1-\sqrt{\frac{8}{i}\left(\ln i+\ln _{2} n\right)}, \quad \text { for } i^{(n)}+1 \leq i \leq n, i^{(n)}=\left\lfloor\alpha_{0} \ln _{2} n\right\rfloor,
$$

with $\alpha_{0}$ large (take $\alpha_{0}>8$ so that $b_{i}^{(n)}>0$ for $n$ large).

This time, we need an extra doubly-indexed, positive sequence $\left(s_{i}^{(n)}\right)_{i=i^{(n)}+1, \ldots, n, n \geq 1}$ such that for $n$ large

$$
\sum_{i=i^{(n)}+1}^{n} s_{i}^{(n)} \leq t_{n}
$$

(Note that this implies $s_{i}^{(n)} \leq 1$.) Similarly, using (6.1) we estimate

$$
\begin{aligned}
& \mathbb{P}\left[S_{n} \leq t_{n}\right] \geq \mathbb{P}\left[\frac{T_{1}^{\prime}}{r^{2\left(U_{1}+\cdots+U_{n}\right)}} \leq s_{n}^{(n)}, S_{2}^{n} \leq t_{n}-s_{n}^{(n)}\right] \\
& \geq \mathbb{P}\left[\frac{T_{1}^{\prime}}{r^{2\left(U_{1}+\cdots+U_{n}\right)}} \leq s_{n}^{(n)}, S_{2}^{n} \leq t_{n}-s_{n}^{(n)}, 2\left(U_{1}+\cdots+U_{n}\right) \geq b_{n}^{(n)} . n\right] \\
& \geq \mathbb{P}\left[T_{1}^{\prime} \leq s_{n}^{(n)} r_{n}^{b_{n}^{(n)} \cdot n}, S_{2}^{n} \leq t_{n}-s_{n}^{(n)}, 2\left(U_{1}+\cdots+U_{n}\right) \geq b_{n}^{(n)} \cdot n\right] \\
& \geq \mathbb{P}\left[T_{1}^{\prime} \leq s_{n}^{(n)} r_{n}^{b_{n}^{(n)} \cdot n}, S_{2}^{n} \leq t_{n}-s_{n}^{(n)}\right]-\mathbb{P}\left[2\left(U_{1}+\cdots+U_{n}\right)<b_{n}^{(n)} \cdot n\right] \\
& \geq \mathbb{P}\left[T \leq s_{n}^{(n)} r_{n}^{b_{n}^{(n)} . n}\right] \times \mathbb{P}\left[S_{n-1} \leq t_{n}-s_{n}^{(n)}\right]-\mathbb{P}\left[2\left(U_{1}+\cdots+U_{n}\right)<b_{n}^{(n)} . n\right] .
\end{aligned}
$$

We iterate the procedure,

$$
\begin{aligned}
\mathbb{P}\left[S_{n-1} \leq t_{n}-s_{n}^{(n)}\right] \geq \mathbb{P}[ & {\left[\leq s_{n-1}^{(n)} \cdot r^{b_{n-1}^{(n)} \cdot(n-1)}\right] \times \mathbb{P}\left[S_{n-2} \leq t_{n}-s_{n}^{(n)}-s_{n-1}^{(n)}\right] } \\
& -\mathbb{P}\left[2\left(U_{1}+\cdots+U_{n-1}\right)<b_{n-1}^{(n)} \cdot(n-1)\right],
\end{aligned}
$$

and so on down to $i^{(n)}$. We obtain

$$
\begin{aligned}
\mathbb{P}\left[S_{n} \leq t_{n}\right] \geq & \left(\prod_{i=i^{(n)}+1}^{n} \mathbb{P}\left[T \leq s_{i}^{(n)} r^{b_{i}^{(n)} . i}\right]\right) \times \mathbb{P}\left[S_{i^{(n)}} \leq t_{n}-\sum_{i=i^{(n)}+1}^{n} s_{i}^{(n)}\right] \\
& -\sum_{i=i^{(n)}+1}^{n}\left(\prod_{j=i+1}^{n} \mathbb{P}\left[T \leq s_{j}^{(n)} r^{b_{j}^{(n)} \cdot j}\right]\right) \times \mathbb{P}\left[2\left(U_{1}+\ldots+U_{i}\right)<b_{i}^{(n)} . i\right] .
\end{aligned}
$$

Using $s_{i}^{(n)} \leq 1$ and $b_{i}^{(n)}<1$, we have, for $n$ large and $i^{(n)}+1 \leq i \leq n$ :

$$
\begin{aligned}
\prod_{j=i+1}^{n} \mathbb{P}\left[T \leq s_{j}^{(n)} r_{j}^{b_{j}^{(n)} \cdot j}\right] & \leq \prod_{j=i+1}^{n} \mathbb{P}\left[T \leq r^{j}\right] \\
& \leq \exp \left(-\sum_{j=i+1}^{n} \mathbb{P}\left[T \geq r^{j}\right]\right) \\
& \leq \exp \left(-\sum_{j=i+1}^{n}\left(\frac{1}{j}-\frac{\ln _{2}(j \ln r)+C}{j^{2} \ln r}\right)\right) \\
& \leq D^{\prime} \frac{i}{n},
\end{aligned}
$$


for some positive constant $D^{\prime}$.

As we did for the series $\sum_{n} a_{n}$, cf. below (6.5) except for using (3.13) instead of (3.12), we easily see that the series $\sum_{n} a_{n}^{\prime}$, with

$$
a_{n}^{\prime}=\sum_{i=i^{(n)}+1}^{n}\left(\prod_{j=i+1}^{n} \mathbb{P}\left[T \leq s_{j}^{(n)} r^{b_{j}^{(n)} \cdot j}\right]\right) \times \mathbb{P}\left[2\left(U_{1}+\ldots+U_{i}\right)<b_{i}^{(n)} \cdot i\right]
$$

is convergent. Now, we choose

$$
s_{i}^{(n)}=\frac{1}{i^{3}},
$$

and we start to bound from below the product

$$
\prod_{i=i^{(n)}+1}^{n} \mathbb{P}\left[T \leq s_{i}^{(n)} r^{b_{i}^{(n)} \cdot i}\right]=\exp \left(\sum_{i=i^{(n)}+1}^{n} \ln \left(1-\mathbb{P}\left[T \geq s_{i}^{(n)} r^{b_{i}^{(n)} i}\right]\right)\right) .
$$

Observe that, by taking $\alpha_{0}>16$, we have $b_{i}^{(n)} \in(1 / 2,1)$ for all large $n$ and $i \in\left[i^{(n)}+1, n\right]$, and also that for large $n$,

$$
\inf \left\{s_{i}^{(n)} r^{b_{i}^{(n)} \cdot i} ; i^{(n)}+1 \leq i \leq n\right\} \geq r^{\frac{\alpha_{0}}{2} \ln _{2} n},
$$

which tends to $\infty$ as $n \rightarrow \infty$. For $i^{(n)}+1 \leq i \leq n$ and $n$ large, in view of (6.9) we have (using $-\ln (1-u) \leq u+u^{2}$ for small $u>0$ and $\frac{1}{1-u} \leq 1+2 u$ for $0<u<\frac{1}{2}$ )

$$
\begin{aligned}
-\ln \left(1-\mathbb{P}\left[T \geq s_{i}^{(n)} r^{b_{i}^{(n)} . i}\right]\right) & \leq \mathbb{P}\left[T \geq s_{i}^{(n)} r^{b_{i}^{(n)} \cdot i}\right]+\varepsilon_{n, i, 1}^{\prime} \\
& \leq \frac{\ln r}{\ln \left(s_{i}^{(n)} r^{b_{i}^{(n)} . i}\right)}+\varepsilon_{n, i, 2}^{\prime} \quad \text { (by (3.4)) } \\
& =\frac{1}{b_{i}^{(n)} \cdot i+\frac{\ln s_{i}^{(n)}}{\ln r}}+\varepsilon_{n, i, 2}^{\prime} \\
& \leq \frac{1}{b_{i}^{(n)} \cdot i}+\varepsilon_{n, i, 3}^{\prime} \\
& \leq \frac{1}{i}+\varepsilon_{n, i, 4}^{\prime},
\end{aligned}
$$

with error terms

$$
\begin{gathered}
\varepsilon_{n, i, 1}^{\prime}=\mathbb{P}\left[T \geq s_{i}^{(n)} r_{i}^{b_{i}^{(n)} . i}\right]^{2}, \quad \varepsilon_{n, i, 2}^{\prime}=\varepsilon_{n, i, 1}^{\prime}+\frac{1}{\ln r} \times \frac{\ln _{3}\left(s_{i}^{(n)} r^{b_{i}^{(n)} \cdot i}\right)+C}{\left(b_{i}^{(n)} \cdot i+\frac{\ln s_{i}^{(n)}}{\ln r}\right)^{2}}, \\
\varepsilon_{n, i, 3}^{\prime}=\varepsilon_{n, i, 2}^{\prime}-2 \frac{\ln s_{i}^{(n)}}{\left(b_{i}^{(n)} \cdot i\right)^{2} \ln r}, \quad \varepsilon_{n, i, 4}^{\prime}=\varepsilon_{n, i, 3}^{\prime}+2 \sqrt{\frac{8}{i^{3}}\left(\ln i+\ln _{2} n\right)} .
\end{gathered}
$$

One can check that $\sup _{n} \sum_{i=i(n)+1}^{n} \varepsilon_{n, i, 4}^{\prime}<\infty$, so for some positive constant $D^{\prime \prime}$, for large $n$,

$$
\begin{aligned}
\prod_{i=i^{(n)}+1}^{n} \mathbb{P}\left[T \leq s_{i}^{(n)} r^{b_{i}^{(n)}} \cdot i\right] & \geq \exp \left(-\sum_{i=i^{(n)}+1}^{n}\left(\frac{1}{i}+\varepsilon_{n, i, 4}^{\prime}\right)\right) \\
& \geq D^{\prime \prime} \frac{i^{(n)}}{n} .
\end{aligned}
$$


Finally, consider the term

$$
\mathbb{P}\left[S_{i(n)} \leq t_{n}-\sum_{i=i^{(n)}+1}^{n} s_{i}^{(n)}\right]
$$

Note that $t_{n}-\sum_{i=i(n)+1}^{n} s_{i}^{(n)}=\frac{\beta}{\ln _{2} n}-\sum_{i=i^{(n)}+1}^{n} \frac{1}{i^{3}} \geq \frac{\beta}{\ln _{2} n}-\frac{1}{2 i^{(n)^{2}}}$, which implies that for all $\beta^{\prime}<\beta, t_{n}-\sum_{i=i(n)+1}^{n} s_{i}^{(n)} \geq \frac{\beta^{\prime}}{\ln _{2} n}$ for large $n$, and then

$$
\mathbb{P}\left[S_{i^{(n)}} \leq t_{n}-\sum_{i=i^{(n)}+1}^{n} s_{i}^{(n)}\right] \geq \mathbb{P}\left[S_{i^{(n)}} \leq \frac{\beta^{\prime}}{\ln _{2} n}\right]
$$

Now, we are ready to conclude the proof: Fix $\alpha_{0}>16$, and let $\beta^{\prime}$ be associated to $\alpha_{0}$ by Lemma 6.3. Then,

$$
\mathbb{P}\left[S_{i(n)} \leq \frac{\beta^{\prime}}{\ln _{2} n}\right] \geq \frac{1}{\ln n},
$$

and for $t_{n}=\left(\beta / \ln _{2} n\right) \wedge 1$ with $\beta>\beta^{\prime}$, using (6.10),

$$
\left(\prod_{i=i^{(n)}+1}^{n} \mathbb{P}\left[T \leq s_{i}^{(n)} r^{b_{i}^{(n)} \cdot i}\right]\right) \times \mathbb{P}\left[S_{i^{(n)}} \leq t_{n}-\sum_{i=i^{(n)}+1}^{n} s_{i}^{(n)}\right] \geq D^{\prime \prime} \frac{i^{(n)}}{n} \times \frac{1}{\ln n} .
$$

Using now (6.8) and $\sum_{n} a_{n}^{\prime}<\infty$ we obtain $\sum_{n \geq 1} \mathbb{P}\left[S_{n} \leq t_{n}\right]=\infty$. By Lemma 6.1 we have a.s.,

$$
T_{n} \leq \frac{\beta A_{n}^{2}}{\ln _{2} n} \quad \text { i.o. }
$$

i.e., $A_{n} \geq \sqrt{\beta^{-1} T_{n} \ln _{2} n}$. Since, for all large $n, \frac{\beta A_{n}^{2}}{\ln 2 n} \leq r_{+}^{n}$, we see that $T_{n} \leq r_{+}^{n}$, so $n \geq \frac{\ln T_{n}}{\ln r_{+}}$, and also

$$
M_{T_{n}}=A_{n} \geq \sqrt{\beta^{-1} T_{n} \ln _{2}\left(\frac{\ln T_{n}}{\ln r_{+}}\right)} .
$$

Finally, for some (small) $K^{\prime}>0$, with probability one, $M_{t} \geq K^{\prime} \sqrt{t \ln _{3} t}$ i.o. The proof of (2.6) is complete.

\section{References}

[1] G. Alsmeyer, D. Buraczewski, A. Iksanov (2017) Null recurrence and transience of random difference equations in the contractive case. J. Appl. Probab. 54, 1089-1110. MR3731286

[2] R. Azencott (1980) Grandes déviations et applications École d'été de Probabilités de SaintFlour 1978, Lect. Notes Math. 774 Springer. MR0590626

[3] M. Babillot, P. Bougerol, L. Elie (1997) The random difference equation $X_{n}=A_{n} X_{n-1}+B_{n}$ in the critical case. Ann. Prob. 25, 478-493. MR1428518

[4] S. Boucheron, G. Lugosi, P. Massart (2013) Concentration inequalities. A nonasymptotic theory of independence. Oxford University Press, Oxford. MR3185193

[5] D. Buraczewski, E. Damek, T. Mikosch (2016) Stochastic models with power-law tails. The equation $X=A X+B$. Cham: Springer MR3497380

[6] A. Cherny (2000) On the strong and weak solutions of stochastic differential equations governing Bessel processes. Stochastics Stochastic Rep. 70, 213-219 MR1800956

[7] F. Comets, S. Popov, M. Vachkovskaia (2016) Two-dimensional random interlacements and late points for random walks. Commun. Math. Phys. 343, 129-164. MR3475663

[8] F. Comets, S. Popov (2017) The vacant set of two-dimensional critical random interlacement is infinite. Ann. Probab. 45, 4752-4785. MR3737923 
[9] F. Comets, S. Popov (2020) Two-dimensional Brownian random interlacement. Potential Analysis 53, 727-771 MR4125109

[10] B. Fristedt, S. Orey (1978) The tail $\sigma$-field of one-dimensional diffusions. Stochastic analysis (Proc. Internat. Conf., Northwestern Univ., Evanston, 1978), pp. 127-138, Academic Press, New York-London. MR0517238

[11] N. Gantert, S. Popov, M. Vachkovskaia (2019) On the range of a two-dimensional conditioned simple random walk. Ann. H. Lebesgue 2, 349-368. MR4015912

[12] M. Gradinaru, Y. Offret (2013) Existence and asymptotic behaviour of some timeinhomogeneous diffusions. Ann. Inst. Henri Poincaré Probab. Stat. 49, 182-207. MR3060153

[13] W. Hoeffding (1963) Probability inequalities for sums of bounded random variables. J. Amer. Statist. Assoc. 58, 13-30. MR0144363

[14] K. Itô, H. P. McKean (1974) Diffusion processes and their sample paths. (2nd. ed.) SpringerVerlag, Berlin-New York. MR0345224

[15] H.G. Kellerer (1992) Ergodic behaviour of affine recursions I: Criteria for recurrence and transience. Techn. report, Univ. München. Available at http://www. mathematik. uni - muenchen . de/ kellerer/I.pdf

[16] D. Khoshnevisan, T. Lewis, W. Li (1994) On the future infima of some transient processes. Probab. Theory Related Fields 99, 337-360. MR1283116

[17] D. Khoshnevisan, T. Lewis, Z. Shi (1996) On a problem of Erdös and Taylor. Ann. Probab. 24 761-787. MR1404527

[18] S. Kochen, C. Stone (1964) A note on the Borel-Cantelli lemma. Illinois J. Math. 8, 248-251. MR0161355

[19] J.C. Pardo (2006) On the future infimum of positive self-similar Markov processes. Stochastics 78, 123-155 MR2241913

[20] S. Popov (2019) Conditioned two-dimensional simple random walk: Green's function and harmonic measure. Journal of Theoretical Probability 34, 418-437 MR4207497

[21] S. Popov (2020) Two-dimensional Random Walk: From Path Counting to Random Interlacements. Cambridge University Press.

[22] S. Popov, L. Rolla, D. Ungaretti, (2020) Transience of conditioned walks on the plane: encounters and speed of escape. Electron. J. Probab. 25, Paper No. 52, 23 pp MR4092771

[23] L.C.G. Rogers (1988) Coupling and the tail $\sigma$-field of a one-dimensional diffusion. Stochastic calculus in application (Cambridge, 1987), 78-88, Pitman Res. Notes Math. Ser. 197, Longman Sci. Tech., Harlow. MR0995472

[24] U. Rösler (1979) The tail $\sigma$-field of time-homogeneous one-dimensional diffusion processes. Ann. Probab. 7, 847-857 MR0542134

[25] D. Stroock, S. Varadhan (2006) Multidimensional diffusion processes. Springer-Verlag, Berlin. MR2190038

[26] A.-S. Sznitman (1998) Brownian motion, obstacles and random media. Springer Monographs in Mathematics. Berlin: Springer. xvi, 353 p. MR1717054

[27] A.-S. Sznitman (2010) Vacant set of random interlacements and percolation. Ann. Math. (2), 171 (3), 2039-2087. MR2680403

[28] A.S. Sznitman (2013) On scaling limits and Brownian interlacements. Bull. Braz. Math. Soc. 44, 555-592 MR3167123

[29] O. Takeyama (1985) Asymptotic properties of asymptotically homogeneous diffusion processes on a compact manifold. J. Math. Soc. Japan 37, 63-650 MR0806305

[30] D. Williams (1974) Path decomposition and continuity of local time for one-dimensional diffusions. I. Proc. London Math. Soc. 28 (3), 738-768. MR0350881

[31] A. Zeevi, P. Glynn (2004) Recurrence Properties of Autoregressive Processes with SuperHeavy-Tailed Innovations. J. Appl. Probab. 41 639-653 MR2074813

[32] M.P. Zerner (2018) Recurrence and transience of contractive autoregressive processes and related Markov chains. Electron. J. Probab. 23, Paper No. 27, 24 pp MR3779820 